\title{
AN INVENTORY OF INDIAN PYRALIDS (LEPIDOPTERA: PYRALIDAE)
}

\section{George Mathew}

Division of Entomology, Kerala Forest Research Institute, Peechi, Kerala 680653, India

Email: mathew@kfri.org

\begin{abstract}
An inventory of 1646 species of pyralids so far recorded from India is presented indicating the year of publication and the current nomenclatural combination. A key adapted from literature is also given for separation of subfamilies.

Kerwords
Checklist, India, inventory, Lepidoptera, moths, Pyralidae,
subfamily key
\end{abstract}

Due to discovery of new species and also due to refinements in the taxonomic techniques, considerable changes have taken place in the taxonomic status of various categories of the Indian Pyralidae since publication of Sir George Hampson's Fauna volume in 1896 in the 'Fauna of British India' series. As a result, the nomenclature of a number of species had undergone changes, some times on several occasions and unaware of these changes, entomologists have been using different names for the same species. For example, the common rice borer, Scirpophaga incertulas (Walker) is also known under the names Tryporiza incertulas and Schoeobius incertulas. Many species originally described under the genus Glyphodes are currently referable under several genera such as Parotis, Palpita, Stemorrhages, Arthroschista etc. Also, no attempt has been made to compile the species so far recorded from India. In addition to the above, a number of species have been published which lie scattered in the literature. A complete taxonomic revision of the family would have been most appropriate but considering the large number of included species and the tremendous task involved, this is not going to be materialised in the immediate future. Until such a revision is made, availability of a list of species occurring in the Indian region will be quite useful for checking the occurrence and nomenclature of species that have been reported from this region.

This check list has been prepared by reference to literature (Arora, 2000; Bleszynski, 1951, 1953, 1961, 1962, 1970; Butler, 1879; Denis \& Schiffermuller, 1775; Guenee, 1854; Hampson, 1896, 1898, 1899a,b,c, 1903, 1906, 1908, 1910, 1912a,b,c,d,e,f,g,h, 1913a,b,c, 1917a,b,c,d,e, 1930; Mathew \& Menon, 1984, 1985, 1986, 1987, 1988; Moore, 1865, 1867, 1879, 1879-88, 1882-87; Munroe \& Mutuura, 1970, Munoroe, 1972, Munroe \& Solis, 1999; Roesler, 1969; Rose, 1981; Rose \& Singh, 1989, 1992, 1999; Snellen, 1882, 1890; Swinhoe, 1884, 1885, 1889a,b, 1892, 1894, 1900, 1906-1907, 1916; and Whalley, 1971). In addition to these, material as well as notes contained on card indexes available in the Natural History Museum, London maintained by the Museum. Full treatment of synonymies and references to nomenclature changes have not been included although the year of publication of the taxa and the current nomenclature combination has been given. Altogether, 1646 species (against 1136 species reported in the Fauna of British India) is given. While every attempt has been made to cover as many species as possible, it is possible that some names could have been left out due to oversight. It is hoped that the present list may serve as a draft for immediate reference until a detailed revision on this group is prepared.

The classification followed in this work is the one proposed by Munroe (1972) for the Pyralidae of America north of Mexico. Munroe and Solis (1999) have also given a detailed taxonomic treatment of this group. A key adapted from the above work is presented here for the separation of various subfamilies.

\section{REFERENCES}

Arora, G.S. (2000). Studies on some Indian pyralid species of economic importance. Part I. Crambinae, Schoenobiinae, Nymphulinae, Phycitinae and Galleriinae (Lepidoptera: Pyralidae). Zoological Survey of India, Kolkata. Records of the Zoological Survey of India, Occasional Paper No. 181, 149pp.

Bleszynski, S. (1951). Studies on the genus Crambus Fb. (Lepidoptera, Crambidae) Polish Society of Entomology, Wroclaw 21: 61-101.

Bleszynski, S. (1953). Studies on the Crambidae (Lepidoptera)-VI. Three new Paleaerctic species of the genus Pediasia Hbn. Annals of Museum of Zoology Poland 15: 101-108.

Bleszynski, S. (1961). A revision of the world species of the family Crambidae, part I - Genus Calmotropha Zell. Acta Zoologica Crac. Krakow 6: $137-272$.

Bleszynski, S. (1962). A short catalogue of the world species of the family Crambidae (Lepidoptera). Acta Zoologica Crac. Krakow 7(12): 197389.

Bleszynski, S. (1970). New genera and species of tropical Crambinae (Studies on Crambinae: Lepidoptera: Pyralidae, pt.48). Tijdschrift voor Entomologie, Deel 113: 1-26.

Butler, A.G. (1879). Illustrations of typical specimens of Lepidoptera Heterocera in the British Museum. Pt. III.

Denis, J.N.C.M. and Schiffermuller (1775). Ankundigungg einen Systematichen Werkes Von den Schmetterlingen der wienergard 1(20): 127146.

Guenee, A. (1854). Species general des Lepidoptera, 8. Deltoides et Pyralites, Paris.

Hampson, G.F. (1896). Fauna of British India, Moths, 4: 1-594. Taylor \& Francis Ltd., London.

Hampson, G.F. (1898). A revision of the moths of sub-family Pyraustinae and family Pyralidae. Part 2. Proceedings of Zoological Society of London 1898: 590-761.

Hampson, G.F. (1899a). A revision of the moths of sub-family Pyraustinae and family Pyralidae. Proceedings of Zoological Society of London 1899: 172-291.

Hampson, G.F. (1899b). The moths of India. Supplementary paper to the volume of "Fauna of British India". Series III., Part III. Journal of the Bombay Natural History Society 1: 164-183, 447-478.

Hampson, G.F. (1899c). The moths of India. Supplementary paper to the volumes of the Fauna of British India" Part IV, V, VI and VIII. Journal of the Bombay Natural History Society 12: 93-98, 304-314, 475-485, 697715 .

(c) Zoo Outreach Organisation; www.zoosprint.org Manuscript 667; Received 16 April 2001; Revised received 22 November 2005; Finally accepted 04 February 2006; Date of publication 21 April 2006 May 2006 | ISSN 0973-2535 (Print edition); 0973-2551 (Online edition) 


\section{Key to subfamilies of Pyralidae}

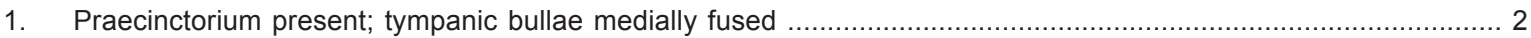

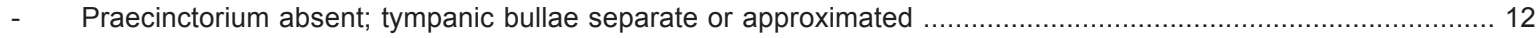

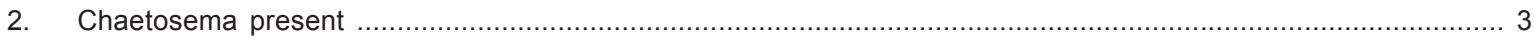

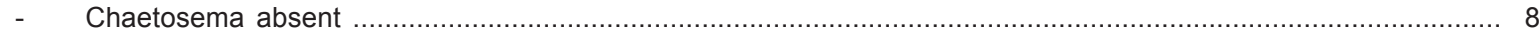

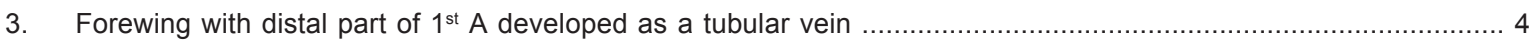

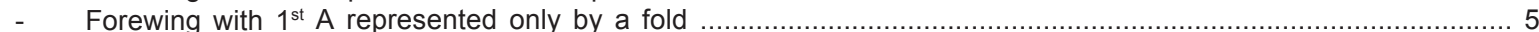

4. Proboscis present; R2 of fore wing stalked with R3+4; M2 of hind wing lost; termen of hind wing usually with a series of black and metallic spots; vinculum of male genitalia without shell-like accessory sclerites Proboacis absent; R2 of fore wing either stalked with or separate from R3+4; M2 of hind wing present; termen of hind wing without a series of black and metallic spots; vinculum of male genitalia with a pair of shell-like accessory selerites

Schoenobiinae

5. Vein R2 of fore wing usually stalked or at least closely apposed to and with R3+4; labial palpus usually upturned and with basal segment relatively long; wings mostly with a conspicuous pattern of transverse bands on a pale ground Nymphulinae (part)

- $\quad$ Vein R2 of fore wing well separated from R3+4; labial palpus porrect, with second segment much longer than basal segment; maxillary palpus having prominent terminal tuft of scales connecting the plane of vertex and frons with dorsal plane of terminal part of labial palpus

6. Forwing usually with weakly raised patches of black scales; cubitus of hind wing usually not distinctly pectinated, with hair-like scales; lateral arms of tegumen of male genitalia about as long as uncus, little tapered ventrally; uncus not strongly compressed or decurved; valvae sometimes with a ventral process but without strong costal or medial armature; gnathos slender and acuminate; the fore-wing pattern almost always resembling that of Noctuidae, in shade of gray or brown powdering ........................................................................................... Scopariinae Forwing without raised patches of black scales; cubitus of hind wing usually distinctly pectinated with hair-like scales, lateral arms of tegumen strongly narrowed ventrally or much longer than uncus, usually both; uncus usually compressed and strongly curved in the vertical plane; gnathos usually long, compressed and decurved; valvae often with strong costal process or medial armature; the fore-wing with longitudinal lines, transverse angular bands near end of cell, silvery areas, black terminal line broken into dot posteriorly, or some combination of these

7. Hind-wing with cell closed, M1 widely separated from Rs Ancylolomiinae (Crambinae) Hindwing with cell open, M1 basally approximated to Rs Crambinae

8. Valvae of male genitalia with costal process; uncus simple; gnathos well developed ...... Cybalomiinae Valvae of male genitalia without costal process, or uncus bi-lobed and laterally decurved and setose, or gnathos absent

Odontiinae

9. Uncus of male genitalia bilobed and laterally setose; gnathos well developed

- Uncus of male genitalia simple or gnathos rudimentary or absent .10

10. Male genitalia with gnathos well developed, slender, dorsally toothed near apex; uncus narrow and distally pointed; valvae usually without clasper, at most with a simple hook-like clasper ................................................ Evergestinae Male genitalia with gnathos almost always weak and rudimentary or absent; gnathos, if well developed, not toothed dorsally and in these species uncus broadly triangular, not long and slender

11. Hind wing with areas of spatulate setae in cubital and anal regions of upperside; praecinctorium simple ....Glaphyriinae Hind wing without areas of spatulate setae in cubital and anal regions above; praecinctorium distally bilobed, often strongly so

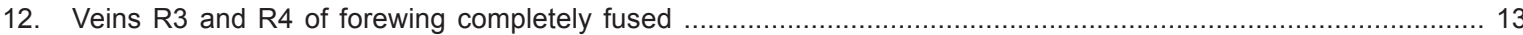

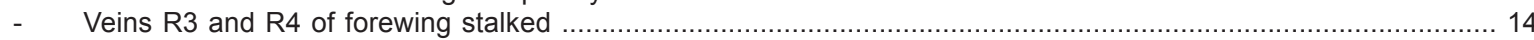

13. Proboscis rudimentary or absent; uncus of male with prominent laterally or postero-laterally directed spine-like processes; ovipositor of female with deep compressed lobes ................................................................ Peoriinae Proboscis usually well developed, sometimes rudimentary or absent;in the latter case, the uncus of the male simple and rounded and the ovipositor of the female with small shallow lobes .............................................. Phycitinae

14. Male genitalia with gnathos prominent, usually slender and distally hooked; uncus various ............................... 15 Male genitalia without gnathos, uncus broad and rounded ............................................................... Galleriinae

15. Maxillary palpi absent; 1 st A of hind wing absent ............................................................................... Chrysauginae

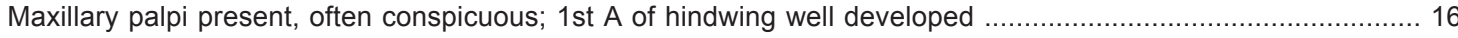

16. Fore wing above tufted with scales; male with labial palpus or basal segment of antenna often produced upward and backward over the vertex; Sc and Rs of the hind wing anastomosed ........................................... Epipaschiinae Fore wing above not tufted with scales; labial palpus and basal segment of antenna not produced upward and

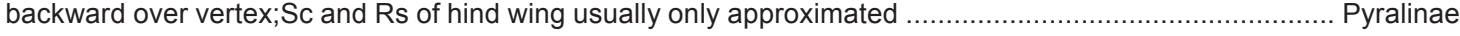




\section{Organisation of the list}

The list is organised in a systematic sequence at supra generic levels although the genera and species are listed alphabetically. Under each genus, the species with author and date of publication are given. In the case of new combinations, the name of the author and the year of publication are given in parenthesis followed by the previous name of the genus, also in parenthesis. A model of the pattern followed in this list is given below:

\section{Galleriinae}

Lamoria Walker, 1863

adaptella (Walker, 1863) (Pempelia)

infumatella Hampson, 1896

\section{List of Pyralidae of India}

\section{Galleriinae}

Achroia Hubner, 1819

grisella (Fabricius, 1794) (Tinea)

innotata (Walker, 1864) (Vobrix)

innotata lankella (Corbet \& Tams, 1943) (Achoria)

Aphomia Hubner, 1825

monochroa (Hampson, 1912) (Melissoblaptes)

odontella (Hampson, 1898) (Melissoblaptes)

vinotincta (Hampson, 1908) (Melissoblaptes)

zelleri De Joannis, 1932 (n.n.bipunctanus Zell.)

(Melissoblaptes)

Cataprosopus Butler 1981

chalybopicta (Warren, 1896) (Lophopalpia)

Corcyra Ragonot, 1885

cephalonica (Stainton, 1866) (Melissoblaptes)

Doloessa Zeller, 1848

castanella (Hampson, 1896) (Thagora)

constellata Hampson, 1898

figurana (Walker, 1863) (Thagora)

ochrociliella (Ragonot, 1893) (Carcinoptera)

Ertzica Walker, 1866

macroptera (Snellen, 1880) (Galleria)

Galleria Fabricius, 1798

mellonella (Linnaeus, 1758) (Tinea)

Lamoria Walker, 1863

adaptella (Walker, 1863) (Pempelia)

hemi Rose, 1951

infumatella Hampson, 1898

jordanis Ragonot, 1998

Megartridia Martin,1956

canosparsalis (Hampson, 1896) (Omphalocera)

Paralipsa Butler, 1879

gularis (Zeller, 1877) (Melissoblaptes)

Prasinoxena Meyrick, 1894

metaleuca Hampson, 1912

Stenachroia Hampson, 1898

elongella Hampson,1898

nigrisparsalis (Hampson, 1903) (Lamoria)

Tirathaba Walker, 1864

grandinotella Hampson, 1898 rosella Hampson, 1898

rufivena (Walker, 1864) (Lamoria)

ruptilinea (Walker, 1866) (Lamoria)

unicolorella (Hampson, 1896) (Mucialla)

Trachylepidia Ragonot, 1887

fructicassiella Ragonot, 1887

\section{Crambinae}

Agriphila Hubner, 1825

hymalayensis Ganey, 1984

impurellus (Hampson, 1895) (Crambus)

tristellus (Denis \& Schiffermuller, 1775) (Tinea)

Ancylolomia Hubner, 1818

argentata Moore, 1886

cervicella Bleszynski, 1970

chrysographellus (Kollar, 1844) (Chilo)

dives Hampson, 1919

felderella Bleszynski, 1970

indica Felder, 1875

intricata Bleszynski, 1970

laverna Bleszynski, 1970

locupletella Kollar, 1844

saundersiella Zeller, 1863

taprobanensis Zeller, 1863

uniformella Hampson, 1895

Bissetia Kapur, 1950

steniellus (Kapur, 1950) (Chilo)

Borer Guenee, 1862

sacchariphagus indicus (Kapur, 1950) (Proceras)

Calamotropha Zeller, 1863

alcesta Bleszynski, 1961

argenticilia (Hampson, 1895) (Crambus)

atkinsoni (Zeller, 1863) (Crambus)

corticellus (Hampson, 1898) (Crambus)

caesella (Walker, 1863) (Araxes)

endopolia (Hampson, 1912) (Crambus)

holodryas (Meyrick, 1933) (Crambus)

indica Bleszynski, 1961

latellus (Snellen, 1890) (Crambus)

lupatus (Meyrick, 1932) (Crambus)

melanosticta (Hampson, 1895) (Crambus)

neurigrammalis (Hampson, 1912) (Crambus)

oculalis (Snellen, 1893) (Crambus)

paludella (Hubner, 1824) (Tinea)

pseudodielota Bleszynski, 1961

punctivenellus (Hampson, 1895) (Crambus)

saturnella Bleszynski, 1961

schwarzi Bleszynski, 1961

venera Bleszynski, 1961

Charltona Swinhoe, 1885

bivitellus Moore, 1872

cervinellus Moore, 1872

consociellus Walker, 1863

cramboides (Walker, 1864) (Lithosia)

desistalis Walker, 1863

diatraeella (Hampson, 1896) (Platytes)

endothermalis Hampson, 1919

fusca Hampson, 1903

inconspicuellus Moore, 1872

kala Swinhoe, 1885

laminata Hampson, 1895

ortellus Swinhoe, 1886

rufalis Hampson, 1919

trichialis (Hampson, 1903) (Platytes)

Charltoniada Strand. 1919

apicella (Hampson, 1896) (Platytes)

Chilandrus Bleszynski, 1970

chrysistes (Meyrick, 1933) (Schoenobius)

Chilo Zincken, 1817 batri (Fletcher, 1927) (Diatraea)

ikri (Fletcher, 1927) (Diatraea)

infuscatellus Snellen, 1890

kanra (Fletcher, 1927) (Diatraea)

partellus (Swinhoe, 1885) (Crambus)

partellus acutus Bhattacherjee, 1971

partellus kanpurensis Bhattacherjee, 1971

sacchariphagus (Bojer, 1873) (Proceras)

sacchariphagus indicus (Kapur, 1950) (Proceras)

suppressalis (Walker, 1863) (Crambus)

tamsi Kapur, 1950

tumidicostalis (Hampson, 1919) (Argyria)

vergilius Bleszynski, 1970

Chilotrea

auricilia (Dudgeon, 1905) (Chilo)

ceylonicus (Hampson, 1895) (Chilo)

polychrysa (Meyrick, 1932) (Diatraea)

Chrysoteuchia (Hubner, 1825)

dividellus (Snellen, 1890) (Crambus)

gonoxes (Bleszynski, 1962) (Crambus)

Classeya Bleszynski, 1960

interstriatellus (Hampson, 1895) (Platytes)

niveifascialis (Hampson, 1895) (Platytes)

Corynophora Berg, 1898

torrentellus (Meyrick, 1879) (Crambus)

Crambixon Bleszynski, 1965

phoeostrigellus (Hampson, 1903) (Crambus)

Crambus Fabricius, 1798

argyrophorus Butler, 1678

athamas Bleszynski, 1961

coryolanus Bleszynski, 1961

dianiphalis Hampson, 1908

hemileucalis Hampson, 1896

nivellus (Kollar,1844)

Culladia Moore, 1866

admigratella (Walker, 1863) (Araxes)

asamella Bleszynski, 1970

dentilinealis Hampson, 1919

suffusella Hampson, 1895

Donagoscaptes Zeller, 1877

aculeata (Hampson, 1903) (Diatraea)

albipennella (Hampson, 1895) (Platytes)

araealis (Hampson, 1912) (Chilo)

belgaumensis (Kapur, 1950 (Coniesta)

diaperalis (Hampson, 1919) (Diatraea)

flavalis (Hampson, 1919) (Diatraea)

forsteri (Bleszynski, 1965) (Acigona)

hampsoni (Kapur, 1950) (Coniesta)

Iunilinealis (Hampson, 1919) (Diatraea)

rufulalis (Hampson, 1919) (Chilo)

steniellus (Hampson, 1898) (Chilo)

strigulalis (Hampson, 1895) (Platytes)

tauromma (Kapur, 1950) (Girdharia)

Epichilo Ragonot, 1888

parvellus Ragonot, 1889

vartianae Bleszynski, 1965

Eschata Walker, 1856

aida Bleszynski, 1970

chrysargyria Walker, 1865

conspurcata Moore, 1888

gelida Walker, 1856

hinalaica Bleszynski, 1965

irrorata Hampson, 1919

mielanacera Hampson, 1896

ochreipes Hampson, 1591

percandida Swinhoe, 1890

rembrandti Bleszynski, 1970

rififi Bleszynski, 1965 
rococo Bleszynski, 1970

xanthocera Hampson, 1895

xanthorhyncha Hampson, 1895

Euchromius Guenee, 1845

ocellea (Haworth, 1811) (Palparia)

Flavocrambus Bleszynski, 1959

melaneurus (Hampson, 1919) (Crambus)

Gargela Walker, 1864

renatusalis (Walker, 1859) (Zebronia)

Girdharia, Kapur, 1950

tauromma Kapur, 1950

Glaucocharis Meyrick, 1938

albilinealis (Hampson, 1896) (Diptychophora)

albilinealis occidentalis Gaskin, 1988

albilinealis similis Gaskin, 1988

assamensis Gaskin, 1988

barmanella Gaskin, 1988

copernici bengalensis Gaskin, 1988

equestris (Meyrick, 1931) (Diptychophora)

fuscopinna Gaskin, 1988

griseolalis (Hampson, 1895) (Diptychophora)

himalayana Gaskin, 1988

iimmitis (Meyrick, 1931) (Diptychophora)

incisella (Bleszynski, 1970) (Paretomene)

kangraensis Gaskin, 1988

khasielia Gaskin, 1988

minutalis (Hampson, 1893) (Ditomoptera)

molleri Gaskin, 1988

ochrophanes (Meyrick, 1931) (Diptychophora)

pilcheri Gaskin, 1988

rectifascialis Gaskin, 1988

rectifascialis indicalis Gaskin, 1988

rusticula (Meyrick, 1931) (Diptychophora)

sikkimella Gaskin, 1988

swanni Gaskin, 1988

tripunctata (Moore, 1888) (Eromene)

tripunctata grahami Gaskin, 1988

tripunctata pallesceni Gaskin, 1988

Libuna Moore, 1886

solitella (Walker, 1866) (Bulina)

Mesolia Ragnot, 1889

margistrigella Hampson, 1899

pandavella Ragonot, 1888

rectilineella Hampson, 1899

Metaeuchromius Bleszynski, 1960

euxonella (Hampson, 1896) (Diptychophora)

mitis (Meyrick, 1931) (Diptychophora)

Microchilo Okano, 1962

acroperalis (Hampson, 1908) (Platytes)

fulvizonella (Hampson, 1896) (Platytes)

Pediasia Hubner, 1825

abbreviatellus (Walker, 1866) (Crambus)

gruberella Bleszynski, 1969

ochristrigellus (Hampson, 1895) (Crambus)

roesleri Bleszynski, 1969

walkeri Bleszynski, 1962

Platytes Guenee, 1845

argyrotricha Hampson, 1934

Prionapteryx Stephens, 1934

albicostalis Hampson, 1919

albipennis (Butler, 1886) (Surattha)

arenalis (Hampson, 1919) (Surattha)

fuscilella (Swinhoe, 1895) (Surattha)

invectalis (Walker, 1863) (Calarina)

scitulella (Walker, 1866) (Surattha)

thysbesalis (Walker 1863) (Pindicitora)
Pseudargyria Okano, 1962

marginepunctalis (Hampson, 1895) (Platytes)

parallelus (Zeller, 1867) (Crambus)

superbella (Zeller, 1863) (Eromene)

Pseudobissetia Bleszynski, 1959

ustalis (Hampson, 1919) (Diatraea)

Pseudocatharylla Bleszynski, 1961

albiceps (Hampson, 1912) (Crambus)

artemida Bleszynski, 1964

chalcipterus (Hampson, 1896) (Crambus)

oenescentellus ceylonica Bleszynski, 1964

Roxita Bleszynski, 1963

adspersella (Snellen, 1893) (Diptchophora)

fletcheri Gaskin, 1984

reductella Gaskin, 1984

Talis Guenee, 1845

cashmirensis (Hampson, 1919) (Diatraea)

Thopeutis Hubner, 1818

galleriellus (Raganot, 1892) (Cephis)

\section{Schoenobiinae}

Brihaspa Moore, 1867

atrostigmella Moore, 1867

Catagela Walker, 1863

adjurella Walker, 1863

Leucoides Hampson, 1893

fuscicostalis Hampson, 1893

Panalipa Moore, 1886

immeritalis (Walker, 1859) (Dosara)

Patissa Moore, 1886

aenealis Hampson, 1899

burmanalis (swinhoe, 1890) (Eurycraspeda)

chlorosema (Meyrick, 1894) (Donacaula)

curvilinealis Hampson, 1895

erythrozonalis (Hampson, 1895)

fulvidorsalis Hampson, 1903

fulvosparsa (Butler, 1881) (Apurima)

interfuscalis Hampson, 1898

intersticalis Hampson, 1908

lactealis (Felder \& Rogenhofer, 1874) (Metasia)

latifuscalis Hampson, 1895

luteifrons Hampson, 1896

multivagellus (Swinhoe, 1886) (Crambus)

Promagrochilo Bleszynski, 1962

ambiguellus (Snellen, 1890) (Chilo)

Ramila Moore, 1867

angustifimbrialis (Swinhoe, 1890) (Crambostenia)

marginella Moore, 1867

ruficostalis Hampson, 1893

Schoenobius Duponchel, 1836

dodatellus (Walker, 1864) (Chilo)

agrophthalmus Meyrick, 1933

niloticus Zeller, 1867

Scirpophaga Treitschke, 1832

auristrigellus (Hampson, 1895) (Schoenobius)

aurivena (Hampson, 1903) (Schoenobius)

bisignatus (Swinhoe, 1885) (Schoenobius)

excerptalis (Walker, 1863) (Chilo)

monostigma Zeller, 1863

flavidorsalis (Hampson, 1919) (Topeutis)

fusciflua Hampson, 1893

gilviberbis Zeller, 1863

incertulas (Walker, 1863) (Chilo)

innotata (Walker, 1863) Tipanaea

khasis Lewvanich, 1981 kuwatai Lewvanich 1981

nepalensis Lewvanich,1981

nivella (Fabricius, 1764) (Tinea)

virginia Schultze, 1908

whalleyi Lewvanich, 1981

xanthogastrella (Walker, 1865) (Apurima)

4. Peoriinae

Baptotropa Hampson, 1918

tricolorella (Hampson, 1899) (Patna)

Emmalocera Ragonot, 1888

anerastica (Snellon, 1880) (Nephopteryx)

aurifusellus (Walker, 1866) (Crambus)

depresella (Swinhoe, 1885) (Melissoblaptes)

endopyrella Hampson, 1918

holorhoda (Hampson, 1908) (Critonia)

lucidicostella Ragonot, 1558

ochracealis (Hampson, 1912) (Critonia)

promelaena (Hampson, 1896) (Nudaria)

purpureotincta (Hampson, 1696) (Critonia)

roseistrigella (Hampson, 1896) (Critonia)

subconcinella (Ragonot, 1890) (Critonia)

umbricostella Ragonot, 1888

umbrivittella (Ragonot, 1888) (Lodiana)

Hypsotropa Zeller, 1848

albivenalis (Hampson, 1912) (Aimballa)

fusifasciata Hampson, 1918

fuscostrigella Ragonot, 1888

heterocerella Hampson, 1896

pervittella Hampson, 1918

punctinervella Hampson, 1918

rosescens Hampson, 1918

Maliarpha Ragonot, 1888

separatella Ragonot, 1888

Metacrateria Hampson, 1918

pulverulella (Hampson, 1896) (Anerastia)

Monoctenocera Hampson, 1899

brachiella Hampson, 1899

Patna Ragonot, 1888

rhizolineata Bradley, 1980

Polyocha Zeller, 1848

depressella (Swinhoe, 1885) (Melissoblaptes)

detritella Ragonot, 1886

diversella Hampson, 1899

ornatella Hampson, 1903

pulverealis Hampson, 1903

strigivenella Hampson, 1899

tricoloralis (Hampson, 1903) (Polyocha)

vesculella Ragonot, 1888

Prophtasia Ragonot, 1887

epiteuxis Hampson, 1918

glaucophaea Hampson, 1918

pyrostrota Hampson, 1918

Raphimetopus Hampson, 1918

ablutella (Zeller, 1839) (Anerastia)

spinifrontella Ragonot, 1886

Rhinaphe Berg, 1875

phaeostrotella Hampson, 1918

Saluria Ragonot, 1887

breviculella Hampson, 1898

discopunctella (Amsel, 1968) (Saluria)

erodella Ragonot, 1888

hemiphaealis (Hampson, 1912) (Saluria)

inficita (Walker, 1863) (Acrobasis)

maculivuttella Ragonot, 1887

minutella Hampson, 1903

nilgiriensis Hampson, 1918 
ochridorsella Ragonot, 1888

opificella (Zeller, 1867) (Anerastia)

paucigraphella Ragonot, 1888

pulverosa (Hampson, 1896) (Poujadia)

rosella Hampson, 1896) (Poujadia)

rufella Hampson, 1918

sepicostella Ragonot, 1888

spurcella Ragonot, 1888

Siboga Hampson, 1901

albimediella Hampson, 1918

dialeucella Hampson, 1918

Tinerastia Hampson, 1901

discipunctella (Hampson, 1896) (Menuthia)

fissirella (Hampson, 1896) (Menuthia)

\section{Phyctinae}

Acrobasis Zeller, 1848

olivalis (Hampson, 1896) (Phycita)

Ammatucha Turner, 1922

semiirrorella (Hampson, 1896) (Nephopteryx)

Ancova Ragonot, 1893

endoleucella Hampson, 1901

flavicollarella (Hampson, 1899) (Rhodophaea)

meridionalis (Walker, 1863) (Nephopteryx)

Ancylodes (Ragonot, 1887)

dunensis Rose, 1981

ilella (Swinhoe, 1884) (Pempelia)

lapsalis (Walker, 1859) (Dosara)

arenaceella (Amsel, 1978) (Ancylosis)

argentescens (Hampson, 1912) (Heterographis)

faustinella (Zeller, 1867) (Euzophera)

fulvimarginella (Hampson, 1903) (Heterographis)

karachiella (Amsel, 1968) (Heterographis)

mysorella (Ragonot, 1888) (Heterographis)

niveicostella (Hampson, 1896) (Ancylosis)

obscuralis (Hampson, 1903) (Heterographis)

octella (Hampson, 1899) (Heterographis)

pakistanella (Amsel, 1968) (Heterographis)

pygmaella (Hampson, 1896) (Heterographis)

resticula (Hampson, 1896) (Heterographis)

rhodochrella Herrich-Schaffer, 1852

microstictella (Hampson, 1903) (Heterographella)

singhalella (Ragonot, 1888) (Heterographis)

trilineatella (Hampson, 1896) (Ancylosis)

umbrilimbella (Ragonot, 1901) (Heterographis)

Anerastia Hubner, 1825

brunneavittella Ragonot, 1888

castanealis Hampson, 1912

celsella (Walker, 1863) (Araxes?)

stictella Hampson, 1908

Anonaepestis Ragonot, 1894

bengalella Ragonot, 1894

Anoristia Ragonot, 1887

venosella (Moore, 1878) (Homoeosoma)

Aphycita Amsel, 1968

sindella Amsel, 1968

Apomyelois Heinrich, 1956

ceratoniae (Zeller, 1939) (Myelois)

Aprophthasia Amsel, 1968

vertianae Amsel, 1968

Asarta Zeller, 1848

alticola (Hampson, 1930) (Anousterunia)

Asemeia Hampson, 1930

aprepia Hampson, 1930
Assara Walker, 1863

albicostalls Walker, 1863

cedrella (Hampson, 1903) (Euzophera)

pinivora (Meyrick, 1933) (Cateremna)

tuberculosa (Meyrick, 1933) (Cateremna)

Auchmera Hampson, 1930

falsalis (Hampson, 1908) (Heterographis)

Aurana Walker, 1863

ctiosaella Walker, 1863

Cadra Walker, 1864

cautella (Walker, 1863) (Pempelia)

Caina Ragonot, 1893

inanitella (Ragonot, 1888) (Anoristia)

Calgula Walker, 1863

defiguralis (Walker, 1863) (Calguia)

Canthelea Walker, 1866

oegnusalis (Walker, 1859) (?Pyralis)

Catastia Hubner, 1825

uniformis (Hampson, 1903) (Etiella)

Cathyalia Ragonot, 1888

fulvella Ragonot, 1888

Caustella Hampson, 1930

micralis (Hampson, 1896) (Heterographis)

Cavipalpia Ragonot, 1893

translucidella Ragonot, 1893

Ceroprepes Zeller, 1867

patriciella Zeller, 1867

pulvillella (Zeller, 1867) (Nephopteryx)

Citripestis Ragonot, 1893

sagittiferella Moore, 1891

pectinicornella (Hampson, 1896) (Myelois)

Conobathra Meyrick, 1886)

atelogramma (Meyrick 1937) (Myelois)

Copamyntis Meyrick, 1934

bipartella (Hampson, 1896) (Phycita)

obliquifasciella (Hampson, 1896) (Phycita)

Crocidophora

phaealis Hampson, 1908

Cryptoblabes Zeller, 1848

angustipennella Ragonot, 1888

ephestialis Hampson, 1903

flavizonalis Hampson, 1912

lophopterella (Hampson, 1899) (Spatulipalpia)

mannheimsi Roesler, 1969

myosticta (Hampson, 1903) (Etiella)

proleucella Hampson, 1896

scotochroalis Hampson, 1912

Ctenomedes Meyrick, 1935

neuractis Meyrick, 1935

Ctenomeristis Meyrick, 1929

ebriola Meyrick, 1934

Cyanaphycis Roesler, 1978

bradleyella Roesler, 1978

ferreotincta (Hampson, 1912) (Nephopteryx)

Didia Ragonot, 1893

strigivenella (Hampson, 1899) (Myelois)

Dioryctria Zeller, 1846

assamensis Mutuura, 1971 castanea Bradley, 1969

raoi Mutuura, 1971

symphoniella (Hampson, 1899) (Phycita)

Eccopidia Hampson, 1899

oinistis Hampson, 1903

strigata Hampson, 1899

Endolasia Hampson, 1896

melanoleuca Hampson, 1896

Ephestia Guenee, 1845

disputella Richards \& Thomson, 1932

glycyphloeas Meyrick, 1935

rubrimediella Hampson, 1896

Ephestiopsis Ragonot, 1893

bipunctalis (Hampson, 1896) (Cryptoblabes)

Epicrocis Zaller, 1848

festivella Zeller, 1848

uberalis (Swinhoe, 1884) (Pyralis)

Epischnia Hubner, 1825

irraralis (Hampson, 1908) (Epischnia)

Ereboenis Meyrick, 1935

saturata Meyrick, 1935

Ernophthora Meyrick, 1887

actiosoides (Hampson, 1896)

Etiella Zeller, 1839

grisea Hampson, 1909

zinckenella (Treitschke, 1832) (Phycis)

Euzophera Zeller, 1867

albicostalis Hampson, 1903

perticella Ragonot, 1888

plumbeifasciella Hampson, 1896

verrucicola (Hampson, 1896) (Heterographis)

Euzopherodes Hampson, 1899

albicans Hampson, 1899

albistrigella Hampson, 1908

dendrophaga Meyrick, 1934

ephestialis Hampson, 1903

hemiphaea Hampson, 1908

irroralis Hampson, 1903

proleucalis Hampson, 1908

taprobalis Hampson, 1908

Gymnancylodes Amsel, 1968

psorosella Amsel, 1968

Heterochrosis Hampson, 1926

zeylanica Hampson, 1926

Homodigma Hampson, 1930

geera Hampson, 1930

Homoeosoma Curtis, 1833

bipunctella Hampson, 1896

ephestidiella Hampson, 1896

fuscella Hampson, 1901

fuscifusella Hampsong 1896

goliathella Ragonot, 1888

griseipennella Hampson, 1896

nigrimedialis Hampson, 1903

punctistrigella Ragonot, 1888

sadhopullensis Rose \& Dhillon, 1980

Hyalospila Ragonot, 1888

leueoneurella Ragonot, 1888

Hydaspia Ragonot, 1888

dorsipunctella Ragonot, 1888 
Hypargyria Ragonot, 1888

ferrella Hampson, 1896

metalliferella Ragonot, 1588

Hypsipyla Ragonot, 1888

elachistalis Hampson, 1903

robusta (Moore, 1886) (Magiris)

rotundipex Hampson, 1903

Idiobrotis Meyrick, 1937

oxygrapha Meyrick, 1937

Indomalayia Roesler \& Kuppers, 1979

flabellifera (Hampson, 1896) (Spatulipalpia)

Indomyrlaea Roesler \& Kuppers, 1979

eugraphella (Ragonot, 1888) (Nephopteryx)

Kaurava Roesler \& Kuppers, 1981

rufimarginella (Hampson, 1896) (Rhodophaea)

Lacipea Walker, 1863

muscosella Walker, 1863

Letoa Walker, 1866

patulella Walker, 1866

Mascelia Hampson, 1930

ectophoea (Hampson, 1908) (Euzophera)

Merulempista Roesler, 1967

Cyclogramma (Hampson, 1896) (Phycita)

Mescinia Hampson, 1901

olivescalis Hampson, 1903

Mesciniodes Hampson, 1901

micans (Hampson, 1896) (Euzophera)

Metallosticha Rebel, 1901

plumbeifasciella (Hampson, 1896) (Euzophera)

Metallostichodes Roesler, 1967

hemicautella (Hampson, 1899) (Euzophera)

Myelois Hubner, 1825

albicostalis Hampson, 1908

atrimaculalis Hampson, 1912

basifuscalis Walker, 1863

echinopisella Chretien, 1911

ephestialis Hampson, 1903

marsysalis Walker, 1863

pectinicornella Hampson, 1896

Nephopteryx Hubner, 1825

acrobasella Hampson, 1912

aeolusalis Walker, 1863

albifascialis (Hampson, 1903)

argiadesalis Walker, 1863

asbolalis Hampson, 1903

basisignella Hampson, 1896

calamalis Hampson, 1903

cyllusalis Walker, 1863

etolusalis Walker, 1863

eugraphella Ragonot, 1888

harpaxalis Walker, 1863

hemiargyralis Hampson, 1908

hypocautella Hampson, 1903

margarophanes Meyrick, 1932

nepheloalis Hampson, 1912

nocturnella Hampson, 1896

ochribasalis Hampson, 1896

rhodobasalis Hampson, 1912

rhyparella Ragonot, 1887

rubralis Hampson, 1903

strigivenata Hampson, 1896

tumidella Hampson, 1903
Oedilepia Hampson, 1930

striginervella (Hampson, 1903) (Nephopteryx)

Oligochroa Ragonot, 1888

leucophaeella (Zeller, 1867) (Pempelia)

majoralis (Hampson, 1896) (Phycita)

pulverulenta (Warren, 1888) (Spermatophthora)

sindella Ragonot, 1893

tenebralls (Hampson, 1896) (Rhodophaea)

flavitinctelia (Ragonot, 1893) (Laodamia)

Pempelia Hubner, 1825

eximielia (Ragonot, 1893) (Salebria)

hemietenis (Meyrick, 1933) (Salebria)

heringii (Ragonot, 1888) (Rhodophaea)

palumbella (Schiffermiiller, 1775) (Tinea)

Phycita Curtis, 1828

ardentia Hampson, 1903

clientella (Zeller, 1867) (Nephopteryx)

clientulella Ragonot, 1893

definalis Hampson, 1908

deodaralis Hampson, 1908

endomelaena Hampson, 1908

erythrolophia Hampson, 1903

eulepidella Hampson, 1896

euzonalis Hampson, 1908

hemipexella Hampson, 1903

hemixanthella Hampson, 1896

infusella (Meyrick, 1879) (Nephopteryx)

jasminophaga Hampson, 1896

melanosticta Hampson, 1912

nodicornella (Ragonot, 1888) (Oligochroa)

ochralis Hampson, 1903

pachylepidella Hampson, 1896

pectenella Hampson, 1896

phaeella Hampson, 1903

steniella Hampson, 1912

umbratalis Hampson, 1912

Phycitodes Hampson, 1917

albatella dierli Roesler, 1973

albovittella (Ragonot, 1888) (Homoeosoma)

nepalensis Roesler, 1973

Plodia Guenee, 1845

interpunctella (Hubner), 1813 (Tinea)

Pristarthria Ragonot, 1893

akbarella (Ragonot, 1888) (Oligochroa)

mundalis (Walker, 1863) (Nephopteryx)

Prorophora Ragonot, 1887

afghanella Roesler, 1973

dialeuca Hampson, 1912

Protoetiella Inoue, 1959

venustella (Hampson, 1896) (Etiella)

Pseudodavara Roesler \& Kuppers, 1979

haemaphoralis (Hampson, 1908) (Spatulipalpia)

Psorosa Zeller, 1846

bifiliferalis Hampson, 1903

proleucalis Hampson, 1903

Ptyomaxia Hampson, 1903

trigonifera Hampson, 1903

Rhodophaea Guenee, 1845

albirenalis Hampson, 1908

nigralbella Hampson, 1899

Sandrabatis Ragonot, 1893

crassiella Ragonot, 1893

phaeella Hampson, 1903

Singhalia Hampson, 1899 cinerea (Hampson, 1896) (Polyocha)

cymogramma (Meyrick, 1933) (Cyphomima)

sarcoglauca (Hampson, 1896) (Critonia)

Spatulipalpia Ragonot, 1893

albicostalis Hampson, 1912

albistrialis Hampson, 1912

effosella Ragonot, 1893

pallicostalis (Walker, 1863) (Nephopteryx)

Staudingeria Ragonot, 1887

bicolorella Roesler, 1973

suboblitelia (Ragonot, 1888) (Heterographis)

Taprobania Hampson, 1930

glaucochroa (Hampson, 1908) (Homoeosoma)

Tephris Ragonot, 1893

connexella Ragonot, 1893

epippella Ragonot, 1890

perlucidella Ragonot, 1893

Thermopteryx Hampson, 1912

rubrifusa Hampson, 1912

Thiallela Walker, 1863

endochralis Hampson, 1908

ligeralis (Walker, 1863) (Myelois)

Thylacoptila Mayrick, 1885

paurosema Meyrick, 1885

Tinestra Hampson, 1908

micralis Hampson, 1908

Trissonca Meyrick, 1882

oxyopa Meyrick, 1934

Vinicia Ragonot, 1893

phloeophaga (Meyrick, 1935) (Eleusina)

Volobilis Walker, 1863

chloropterella (Hampson, 1896) (Phycita)

Zynodes Whalley, 1970

strigerella (Nampson, 1903) (Blabiodes)

\section{Epipaschiinae}

Coenodomus Walsingham, 1889

dudgeoni Hampson, 1896

fumosalis Hampson, 1903

hockingi Walsingham, 1889

rotundinidus Hampson

Jocara Walker, 1863

malefica (1934)

rufescens (Hampson, 1896) (Stericta)

Lamida Walker 1858

mediobarbalis (Hampson, 1916) (Macalla)

moncusalis Walker, 1858

sordidalis (Hampson, 1916) (Macalla)

Lepidogma Meyrick, 1890

chlorophilalis Hampson, 1912

melanolopha Hampson, 1912

minimalis (Hampson, 1916) (Pococera)

olivalis (Swinhoe, 1895) (Hypsopygia)

rufescens Hampson, 1896

tamaricalis (Mann., 1873) (Hypotia)

Lista Walker, 1859

ficki (Christ., 1881) (Craneophora)

variegate (Moore, 1879) (Scopocera)

Locastra Walker, 1858

crassipennis (Walker, 1857) (Eurois)

pachylepidalis Hampson, 1896 
Magalla Walker, 1859

albifurcalis Hampson, 1916

atricinctalis Hampson, 1916

brachyscopalis Hampson, 1912

carbonifera (Meyrick, 1932)

cuproviridalis Moore, 1867

dimidialis (Snellen, 1890) (Pannucha)

eumictalis Hampson, 1912

exrufescens Hampson, 1896

hypnonalis Hampson, 1899

hypoxantha Hampson, 1896

mesoleucalis Hampson, 1916

vetasarcia Hampson, 1903

nubilalis (Hampson, 1893) (Stericta)

ochroalis Hampson, 1916

parvula Hampson, 1896

phaeoperalis Hampson, 1916

plicatalis Hampson, 1903

rufibarbalis Hampson, 1903

rufitinctalis (Warren, 1896) (Parasarama)

scoporhyncha Hampson, 1896

Noctuides Staudinger, 1892

melanophia (Staudinger,1892) (Anartula)

thurivora (Meyrick, 1932) (Anartula)

Odontopaschia Hampson, 1903

virescens Hampson, 1903

Omphalota Hampson, 1899

chlorobasis Hampson, 1899

Orthaga Walker, 1859

aenescens (Moore,

auroviridalis Hampson, 1896

basalis Moore

euadrusalis Walker, 1858

euryzona Hampson, 1896

exvinacea (Hampson, 1891) (Balanotis)

irrorata Hampson

leucatma (Moyrick, 1932) (Balanotis)

mangiferae Misra, 1932

molleri Hampson, 1896

onerata Butler

rhodoptila (Meyrick, 1932) (Balanotis)

roseiplaga Hampson, 1896

rudis (Walker, 1862) (Locastra)

vitialis (Walker, 1859) (Pyralis)

Peplochora Meyrick, 1932

zalalges Meyrick, 1933

Stericta Lederer, 1863

aglossalis (Warren, 1896) (Scopocera)

asopialis (Snellen, 1890) (Pannucha)

atkinsoni Moore

callibrya Meyrick, 1933

carneotincta Hampson, 1896

divitalis Guenee

olivalis Hampson, 1903

plumbifloccalis Hampson, 1896

pyraliata (Moore, 1888) (Scopocera)

rubrescens Hampson, 1903

sinuosa Moore,

trichasema Hampson, 1916

Teliphasa Moore, 1888

albifusa (Hampson, 1896) (Macalla)

Trichotophysa Warren, 1896

jucundalis (Walker, 1865) (Stericta)

7. (1). Pyralinae (Endotrichini)

Endotricha Zeller, 1847

albicilia Hampson, 1891

ardentalis Hampson, 1896

costaemaculalis Christoph, 1881

fuscifusalis Hampson, 1896 decessalis Walker, 1859

eximia Whalley, 1963

fuscobasalis Ragonot, 1891

loricata Moore, 1888

luteogrisalis Hampson, 1896

melanobasis Hampson, 1916

mesenterialis Walker, 1859

7. (2). Pyralinae (Pyralini)

Aglossa Latreille, 1796

pinguinalis indistincta Corbet \& Tams, 1943

tanya Corbet and Tams, 1943

Arctioblepsis Felder \& Felder, 1862

rubida Felder, 1862

Bostra Walker, 1863

angulifacia (Moore, 1888) (Pyralis)

arida (Butler, 1881) (Rhodaria)

astigma Hampson,

balux (Swinhoe, 1884) (Micra)

castanoptera Moore

denticulata Swinhoe, 1890

fascialis (Warren, 18951 (Orthopygia)

ferrifusalis Hampson, 1899

flammalis Hampson, 1906

flavilinealis Hampson, 1906

gnidusalis (Walker, 1859) (Aglossa)

tristis (Butler, 1881) (Scotomera)

igneusta Swinhoe

ignirubralis (Hampson, 1917)

illusella Walker, 1863

indicator (Walker), (Arippana)

imperatrix (Warren, 1896) (Zitha)

loxotona Meyrick, 1933

maculilinea Hampson, 1917

mesoleucalis Hampson, 1912

metaxanthialis Hampson, 1906

nephelorthra Meyrick, 1933

orthocopa Meyrick, 1937

pallidicosta (Hampson, 1893) (Stemmatophora)

ruptilinealis (Warren, 1895) (Comara)

salmo Hampson

sarcosia Hampson, 1912

subviridescens (Warren, 1895) (Orthopygia)

vibicalis(Lederer, 1863) (Bostra)

Crocalia Ragonot, 1891

aglossalis Ragonot, 1891

Dattinia Ragonot, 1887

ereminalis (Swinhoe, 1889) (Surratha)

opiparalis (Swinhoe, 1889) (Zonara)

proximalis (Christoph, 1882) (Hypotia)

rubella (Swinhoe, 1884) (Hypotia)

vulgaris (Butler, 1881) (Hypotia)

Discordia Swinhoe, 1885

basalis Hampson, 1896

evulsa Swinhoe, 1885

siniferalis Hampson, 1896

Embryoglossa Warren, 1896

bipuncta Hampson, 1903

variegata Warren,1896

Euryzonella Ghesquiere, 1942

latisfascia (Hampson, 1891) (Pyralis)

Herculia Walker, 1859

albolinealis (Hampson, 1891) (Pyralis)

aurocilialis Hampson, 1891

bractealis Walker, 1859

dharmsalae Butler, 1889

flammealis Hampson, 1906

fuscalis Hampson

hansi Rose \& Dhillon, 1980

ignifualis (Walker, 1859) (Pyralis) imbecilis (Moore, 1885) (Pyralis)

marthalis (Walker, 1859) (Pyralis)

mediallis Hampson, 1903

incongrua (Butler, 1886) (Pyralis)

ochreicilia Hampson, 1891

purpureorufa Hampson, 1917

rudis (Moore, 1888) (Stemmatophora)

sericea (Warren, 1891) (Hypsopygia)

suffusalis (Walker, 1865) (Pyralis)

Heterocrasa Warren, 1896

expansalis Warren, 1896

Hyboloma Ragonot, 1891

pallidalis Hampson, 1906

Hypsopygia Hubner, 1825

postflava (Hampson, 1893) (Pyralis)

Lamacha Walker, 1863

angulifera Hampson, 1903

Larice Ragonot, 1891

swinhoei Ragonot, 1891

Loryma Walker, 1859

recusata (Walker, 1863) (Beria)

Omphalota Swinhoe, 1894

accersita Swinhoe, 1894

Orybina Snellen, 1894

flaviplaga (Walker, 1863) (Oryba)

plangonalis (Walker, 1859) (Scopula)

Paractenia Ragonot, 1891

castaneonigra Hampson, 1917

pallidirubra Hampson, 1917

pallucidalis Warren

quisqualis Swinhoe, 1885

rubicundalis Swinhoe, 1885

semiochrea (Warren, 1895) (Stemmatophora)

sichimensis Hampson, 1917

Polycampsis Warren, 1896

longinasus (Warren, 1896) (Polycampsis)

Proropera Warren, 1896

vinosalis Warren, 1896

Prosaris Meyrick, 1894

pernigralis Meyrick, 1894

Proteinia Hampson, 1896

pailifrons (Snellen, 1890) (Toccolosida)

Pyralis Linnaeus, 1758

elongalis (Kollar, 1844) (Asopia)

fumipennis Butler, 1889

funebralis Warren, 1895

janiusalis Walker, 1859

manihotalis Guenee, 1854

palesalis Walker, 1859

phycidalis Guenee, 1854

proximalis Walker, 1862

pictalis (Curtis, 1834) (Asopia)

prepialis Hampson, 1903

ravolalis Walker, 1859

recisalis Swinhoe, 1885

regalis Denis \& Schiffermuller, 1775

subresectalis (Snellen, 1890) (Asopia)

Rhynchetera Hampson, 1896

carnealis Hampson, 1896

Rhynchopygia Hampson, 1896

purpureorufa Hampson, 1896 
Rostripalpus Hampson, 1896

longipennis Hampson, 1896?

Sacada Walker, 1862

constrictalis (Ragonot, 1891) (Sybrida)

discinota (Moore, 1866) (Paravetta)

flexuosa (Snellen, 1890) (Paravetta)

inordinata (Walker, 1865) (Sybrida)

pallescens (Hampson, 1896) (Sacada)

pyraliformis (Moore, 1879) (Danaka)

rufina (Hampson, 1896) (Sacada)

sikkima (Moore, 1879) (Paravetta)

unilinealis (Hampson, 1896) (Sacada)

Stemmatophora Guenee, 1854

costinotatalis Hampson, 1906

fuliginalis Hampson, 1906

fuscibasalis (Snellen, 1880) (Stericta)

herculialis Hampson, 1896

laticincta Hampson, 1896

minimalis Hampson, 1917

minoralis Hampson, 1903

monostaechalis Warren

pallidella Hampson, 1896

pseudaglossa Hampson, 1896

punctimarginalis Hampson, 1896

rectisectalis (Warren, 1896) (Pyralis)

rivulata Moore

sanguifusa Hampson, 1896

scotalis Hampson, 1906

subflavalis Hampson, 1903

tactilis Swinhoe, 1890

valida Butler

Synaphe Hubner, 1825

indica Strand. 1914

Tamraca Moore, 1887

moorei Rose \& Pajni, 1978

torridalis (Lederer, 1863) (Asopia)

Tegulifera Saalmuller, 1879-80

bicoloralis (Leech, 1889) (Stemmatophora)

castanealis Hampson, 1896

drapesalis (Walker, 1859) (Pyralis)

fumiosalis (Warren, 1896) (Peucela)

pallidibasalis Hampson, 1896

phaeaptera Hampson, 1903

psamathopis (Meyrick, 1894) (Herculia)

rosealis Hampson, 1896

rufifascialis (Hampson, 1896)

subolivescens (Warren, 1895) (Orthopygia)

tripartita Hampson, 1917

Teratomorpha De Niceville, 1896

hampsoni De Niceville, 1896

Toccolosida Walker, 1863

rubriceps Walker, 1863

Trebania Ragonot, 1891

muricolor Hampson, 1896

Triphassa Hubner, 1818

bilineata Moore, 1878 (Herculia)

imbutalis Walker, 1866 (Rhodaria)

lutecilialis Hampson, 1896

macrarthralis Hampson, 1908

aedalis (Hampson, 1912 (Stemmatophora)

metaxantha Hampson, 1896

ochrealis Hampson, 1893

vulsalis (Walker, 1859) (Botys)

xylinalis Swinhoe, 1885

zeuxoalis (Walker, 1863) (Pindicitora)

zonalis Hampson, 1899

Tyndis Ragonot, 1891

albidefinis Hampson, 1903 erebalis Hampson, 1899

hypotialis Swinhoe, 1886

plana (Walker, 1862) (Anthophila)

Vitessa Moore, 1860

suradeva Moore, 1860

suradeva rama Moore, 1885

Xenomilia Warren, 1896

humeralis Warren, 1896

\section{Musotiminae}

Cymoriza Guenee, 1854

irrectalis Guenee, 1854

ustalis Walker, 1865

Musotima Meyrick, 1884

acclaralis (Walker, 1859) (Isopteryx)

colonalis (Bremer, 1864) (Ambia)

instrumentalis (Swinhoe, 1894) (Ambia)

suffusalis (Hampson, 1893) (Ambia)

Uthinia Snellen, 1898

albisignalis (Hampson, 1896) (Orphnophanes)

\section{Nymphulinae}

Agassiziella Yoshiyasu, 1989

albidivisa (Warren, 1896) (Oligostigma)

angulipennis (Hampson, 1891) (Oligostigma)

fuscifusalle (Hampson, 1893) (Oligostigma)

picalis (Guenee, 1854) (Oligostigma)

Ambia Walker, 1859

albipunctalis Warren, 1896

caeruleata Hampson, 1893

complicata Warren

conspurcatalis Warren, 1896

iambesalis Walker, 1859

lobophoralis Hampson, 1896

marginalis Moore

poritialis Walker, 1859

semifascialis Warren, 1896

tenebrosalis Hampson, 1896

xantholcuca Hampson, 1896

Aulacodes Guenee, 1854

colonialis Guenee

dominalis Walker, 1865

hamalis Snellen

saturatalis (Snellen, 1890) (Oligostigma)

Camptomastix Warren, 1892

exuvialis (Snellen, 1890) (Diplotyla)

hisbonalis (Walker, 1859) (Botys)

Cataclysta Hubner, 1827

angulata Moore

trimacula Hampson

Clupeosoma Snellen, 1880

sericialis (Hampson, 1896) (Pleonectoides)

Elophila Hubner, 1822

melagynalis (Agassiz, 1978) (Nymphula)

Eoophyla Swinhoe, 1900

melanops (Hampson, 1896) (Aulacodes)

peribocalis (Walker, 1859) (Cataclysta)

sejunctalis (Snellen, 1876) (Oligostigama)

Eristena Warren, 1896

bifurcalis (Pryer, 1877) (Oligostigma)

murinalis (Warren, 1896)

oligostigmalis Hampson, 1906

postalbalis Hampson

pulchellalis (Hampson) (Oligostigma)

straminealis Hampson, 1903
Goniopalpia Hampson, 1903

delicatalis Hampson, 1903

Nymphicola Snellen, 1880

blandialis (Walker, 1859) (Cataclysta)

Nymphula Schrank, 1802

affinialis (Guenee, 1854) (Parapoynx)

falliolatalis Swinhoe, 1890

fusalis Hampson, 1896

fuscicostalis Hampson, 1896

grisialis Hampson, 1912

leucostola Hampson, 1896

pygmaealis Warren, 1896

responsalis (Walker, 1865) (Diasemia)

votalis (Walker, 1854) (Oligostigma)

Oligostigma Guenee, 1854

albifurcalis Hampson, 1906

alicialis Hampson, 1906

andreusialis Hampson, 1912

araealis Hampson, 1897

auropunctalis Hampson, 1903

chrysozonalis Hampson, 1912

dianalis Hampson

excisa (Swinhoe, 1901) (Hemiloba)

fumibasalis Hampson, 1896

hapilista Swinhoe, 1892

melanotalis Hampson, 1906

niveinotatumt Hampson

ornatum Moore

parvalis Moore, 1877

Paracataclysta Yoshiyasu, 1983

fuscalis (Hampson, 1893) (Cataclysta)

Paracymoriza Warren, 1890

albifascialis (Hampson) (Parthenodes)

aurantialis (Swinhoe) (Parthenodes)

exsolvalis (Snellen, 1882) (Hydrocampa)

inextricata (Moore, 1888) (Cymoriza)

vagalis (Walker, 1865) (Parthenodes)

Parapoynx Huebner, 1825

bilinealis (Snellen, 1876) (Oligostigma)

crisonalis (Walker, 1859) (Hydrocampa)

diminutalis Snellen, 1880

fluctuosalis (Zeller, 1852) (Nymphula)

stagnalis (Zeller, 1852) (Nymphula)

Parthenodes Guenee, 1854

aequivocalis Warren, 1896

bisangulata (Hampson, 1895) (Brihaspa)

gangeticalis (Lederer, 1863) (Parapoynx)

latifascialis Warren, 1896

nigra (Warren, 1896) (Nymphula)

nigriplaga Swinhoe

olivalis Hampson

stellata (Warren, 1896) (Paracymoriza)

Strepsinoma Meyrick, 1897

croesusalis (Walker, 1859) (Cataclysta)

Teratausta Hampson, 1903

odontalis Hampson, 1903

10. Scopariinae

Dasyscopa Meyrick, 1894

homogenes Meyrick, 1894

Heliothela Guenee, 1854

ophideresana (Walker, 1863) (Orosana)

Micraglossa Warren, 1891

distictalis Hampson, 1908)

oenealis Hampson, 1897

scoparialis Warren, 1891 
Scoparia Haworth, 1811 albifusalis Hampson, 1906 canicostalis Hampson, 1896 crocalis Hampson, 1903 delicatalis Walker, 1865 medinella Snellen, 1890 mediorufalis Hampson, 1896 murificalis Walker, 1859 ochrotalis Hampson, 1903 olivaris Hampson, phaealis Hampson, 1903 polialis Hampson, 1903 pulveralis Snellen, 1890 rufostigma Hampson straminealis Hampson, 1903 vinotinctalis Hampson, 1896

\section{Odontinae}

Autocmaris Swinhoe, 1874

fessalis (Swinhoe, 1886) (Glyphodes)

margaronialis Hampson, 1912

moringae Tams, 1938

Baeotarcha Meyrick, 1884 hyalinalis Hampson, 1896 martinalis Walker, 1859

Cheloterma Meyrick, 1933 invidiosa Meyrick, 1933

Dausara Walker, 1859 talliusalis Walker, 1859

Hemiscopis Warren, 1890 expansa Warren, 1892 suffusalis (Walker, 1865) (Scopula)

Heortia Lederer, 1863 vitessoides (Moore, 1885) (Tyspana)

Irigilla Swinhoe, 1900 purpuralis (Hampson, 1908) (Calamochrous)

Noctuelia Guenee, 1854 dichroalis Hampson, 1903 floralis (Hubner, 1809) (Pyralis)

Porphyronoorda Munroe, 1977 decumbens Munroe, 1977

Taurometopa Meyrick, 1933 aryrostrota (Hampson, 1917) (Balaenifrons)

Tegostoma Zeller, 1847 baphiales (Staudinger, 1870) (Anthophilodes) flavida Moore, 1881

Titanio Hubner, 1829

leucopsumis (Hampson, 1919) (Argyria)

\section{Evergestiinae}

Crocidolomia Zeller, 1852

luteolalis Hampson, 1893

pavonana (Fabricius, 1794) (Pyralis)

suffusalis (Hampson, 1891) (Godara)

13. Glaphyriinae

Hellula Guenee, 1854

undalis (Fabricius, 1781) (Phalaena)

reniculus Pajni \& Rose, 1977

Thyridiphora Warren, 1888

furia (Swinhoe, 1884) (Micra)

\section{Cybalomiinae}

Cybalomini Marion, 1959

Cybalomia Lederer, 1863

cervinalis Hampson, 1908
Hendecasis Hampson, 1896

duplifascialis (Hampson, 1891) (Trichophysetis)

fulvalis Hampson

minutalis Hampson, 1906

Ptychopseustis Meyrick, 1889

amoenella (Snellen, 1880) (Diptychophora)

argentisparsalis (Hampson, 1895) (Platytes)

fuscivenalis (Hampson, 1895) (Platytes)

ictericalis (Swinhoe, 1885) (Platytes)

plumbeolinealis (Hampson, 1895) (Platytes)

undulalis (Hampson, 1919) (Argyria)

Trichophysetis Meyrick, 1884

acutangulalis Hampson, 1903

fumilauta (Warren, 1896) (Hendecasis)

gracilentalis Swinhoe, 1890

metamelalis Hampson, 1899

nigricincta Hampson

nigridiscalis Warren, 1895

nigripalpis (Warren, 1896) (Callinaias)

pygmaealis (Warren, 1896) (Endotricha)

rufoterminalis (Christ, 1881) (Endotricha)

simplalis (Snellen, 1890) (Hydrocampa)

umbrifusalis Hampson, 1912

\section{Pyraustinae}

Achyra Guenee, 1849

coelatalis (Walker, 1859) (Dosara)

nudalis (Hubner, 1796) (Pyralis)

Acropentias Meyrick, 1890

pulverulenta (Warren, 1892) (Ebulea)

Aethaloessa Lederer, 1863

calidalis (Guenee, 1854) (Glyphodes)

floridalis (Zeller, 1852) (Stenia)

Aetholix Lederer, 1863

flavibasalis (Guenee, 1854) (Aediodes)

indecisalis Warren, 1896

Agastya Moore, 1881

hyblaeoides Moore, 1881

Agathodes Guenee, 1854

modicalis Guenee, 1854

monstralis Guenee, 1854

ostentalis (Geyer, 1837) (Perinephela)

Agrioglypta Meyrick, 1932

eurytusalis (Walker, 1859) (Glyphodes)

itysalis (Walker, 1859) (Glyphodes)

naralis (Felder \& Rogenhofer, 1875) (Glyphodes)

zelimalis (Walker, 1859 (Glyphodes)

Agrotera Schrank, 1802

barcealis (Walker, 1859) (Leucinodes)

basinotata Hampson, 1891

coelatalis (Walker, 1859) (Nistra)

discinotata Swinhoe

effertalis Walker, 1859

leucostola Hampson, 1896

magnificalis Hampson

scissalis Walker, 1865

Almonia Walker, 1865

cristata (Hampson, 1891) (Dodanga)

lobipennis Moore

truncatalis Walker, 1865

Analyta Lederer, 1863

melanopalis Guenee, 1854

sigulalis (Guenee, 1854) (Leucinodes)

Anamalaia Munroe \& Mutuura, 1969

nathani Munroe \& Mutuura 1969
Anania Hubner, 1825

verbascalis (Denis \& Schiffermuller, 1775) (Pyralis)

Antigastra Lederer, 1863

catalaunalis (Duponchel, 1833) (Botys)

Archernis (Meyrick, 1886)

capitalis (Fabricius, 1794) (Phalaena)

dolopsalis (Walker, 1859) (Botys)

fulvalis Hampson, 1899

humilis Swinhoe

lugens (Warren, 1896) (Protonoceras)

nictitans Swinhoe

obliquialis Hampson, 1896

Aristebulea Munroe \& Mutuura, 1968

nobilis Moore

Arthroschista Hampson, 1893

hilaralis (Walker, 1859) (Margaronia)

Auchmophoba Turner, 1913

alternata (Warren, 1895) (Petta)

costistrigalis (Hampson, 1896) (Petta)

Aulacoptera Hampson, 1896

fuscinervalis (Swinhoe, 1895) (Aulacophora)

Banepa Moore, 1887

atkinsoni Moore, 1887

Bocchoris Moore, 1885

acamasalis (Walker, 1859) (Zebronia)

adipalis (Lederer, 1863) (Botys)

albinalis Hampson, 1912

aptalis (Walker, 1865) (Botys)

artificalis (Lederer, 1863) (Botys)

ciliata Swinhoe

clathralis (Swinhoe, 1894) (Ceratarcha)

danalis Hampson

incisalis (Snellen, 1880) (Botys)

inspersalis (Zeller, 1852) (Botys)

pusaensis (Bhattacherjee, 1973) (Hymenia)

onychinalis (Guenee, 1854) (Asopia)

rotundalis (Hampson, 1893) (Synclera)

sphenocosma (Meyrick, 1894) (Margaronia)

telphusalis (Walker, 1859) (Glyphodes)

tenera (Butler, 1883) (Hydrocampa)

trimaculalis (Snellen, 1880) (Aediodes)

trivitralis Swinhoe, 1895

Botyodes Guenee, 1854

asialis Guenee, 1854

caldusalis Walker, 1859

crocopteralis Hampson, 1898

flavibasalis Moore, 1867

patulalis Walker, 1865

principalis Leech, 1889

Bradina Lederer, 1863

adhaesalis Walker, 1858

admixtalis (Walker, 1859) (Botys)

diagonalis (Guenee, 1854) (Stegothyris)

melanoperas Hampson, 1896

subpurpurescens Warren, 1896

translinealis Hampson, 1896

Calamochrous Lederer, 1863

bipunctalis Hampson, 1912

carnealis (Swinhoe, 1895) (Notaspis)

dichroma (Moore, 1879) (Ebulea)

ferruginalis Hampson, 1896

homochroalis Swinhoe, 1907

pentasaris Meyrick, 1932

ruficostalis Hampson, 1896

sarcalis Hampson, 1908

sterrhalis Hampson, 1903 
Cadarena Moore, 1886

sinuata Fabricius,

Callibotys Munroe \& Mutuura, 1969

hyalodiscalis (Warren, 1895) (Rhectothyris)

Cangetta Moore, 1886

albocarnea Warren, 1896

homoperalis Hampson, 1899

Caprinia Walker, 1859

felderi Lederer, 1863

intermedia Warren, 1896

Ceratarcha Swinhoe, 1894

umbrosa Swinhoe, 1894

Chalcidoptera Butler, 1887

appensalis Snellen, 1884

emissalis (Walker, 1866) (Botys)

rufilinealis Swinhoe, 1895

Chrysot hyridia Munroe, 1967

invertalis (Snotien, 1877) (Bocchoris)

Circobotys Butler, 1879

malaisei Munroe \& Mutuura, 1970

Cirrhochrista Lederer, 1863

bracteolalis Hampson, 1891

brizoalis (Walker, 1859) (Margaronia)

fumipalpis Felder, 1874

pulchellalis Lederer, 1863

semibrunnea Hampson, 1896

spissalis (Guenee, 1854) (Botys)

Cnaphalocrocis Lederer, 1863

medinalis (Guenee, 1854) (Salbia)

Coelorhyncidia Hampson, 1896

ovulalis Hampson, 1896

Coptobasis Lederer, 1863

arctalis (Guenee, 1854) (Sylepta)

Cotachena (Moore, 1885)

histricalis (Walker, 1859) (Botys)

whalleyi Rose \& Pajni, 1977

Crocidophora Lederer, 1863

acutangulalis Swinhoe,

amoenalis Snellen, 1890

aurimargo (Warren, 1896) (Circobotys)

calvatalis Swinhoe, 1890

curvilinealis Hampson, 1899

discolorata Swinhoe

distinctalis Swinhoe, 1899

exstigmalis Hampson, 1903

fasciata Moore

flavofasciata Moore, 1879

fulvidalis Warren, 1895

fuscalis Hampson

griseifusa Swinhoe, 1891

limbata Moore

lutusalis (Snellen, 1890) (Crocidophora?)

multidentalis Warren, 1895

pallida Moore

pallidulalis Swinhoe

pionealis (Snellen, 1890) (Bradina)

ptyophora Hampson, 1896

sinisalis Walker, 1859

stenophilalis (Walker, 1865) (Botys)

Crypsiptya Meyrick, 1894

coclesalis (Walker, 1859) (Botys)

mutuuri (Rose \& Pajni, 1979) (Coclebotys)

Cydalima Lederer, 1863

capriniodes (Hampson, 1912) (Glyphodes) conchylalis (Guenee, 1854) (Margarodes)

laticostalis Guenee, 1854

Daulia Walker, 1859

afralis Walker

argyrostrotalis Hampson, 1912

aurantialis Hampson, 1896

Diathrausta Lederer, 1863

ochrifuscalis (Hampson, 1903) (Endotricha)

picata (Butler, 1889) (Danaga)

profundalis (Lederer, 1863) (Diathrausta)

Diathraustodes Hampson, 1896

leucotrigona Hampson, 1896

similis Hampson, 1903

Deanolis Snellen, 1899

albizonalis (Hampson, 1903) (Noorda)

Diaphania Hubner, 1825

indica (SaundersI1851) (Eudioptes)

lacustralis Moore, 1867

perspectalis (Walker, 1859) (Phakellura)

Diasemia Hiibner, 1825

accalis (Walker, 1859) (Scopula)

reticularis (Linnaeus, 1761) (Pyralis)

Diasemiopsis Munroe, 1957

ramburialis (Duponchel, 1834) (Hydrocampa)

Dichocrocis Lederer, 1863

actinialis Hampson, 1898

atrisectalis Hampson, 1908

attemptalis (Snellen, 1890) (Botys)

bistrigalis Walker, 1865

credulalis (Snelien, 1890) (Botys)

definita (Butler, 1889) (Haritala)

dehradunensis (Pajni \& Rose, 1977)

diminutive (Warren, 1696) (Conogethes)

evaxalis (Walker, 1859) (Botys)

festivalis Swinhoe, 1885

haemactalis (Snellen, 1890) (Conogethes)

klotsi Pajni \& Rose, 1977

leptalis Hampson, 1903

macrostidza Hampson, 1912

megillalis (Walker, 1859) (Botys)

nilusalis (Walker, 1859) (Botys)

nigrilinealis (Walker, 1866) (Botys)

pandamalis (Walker, 1859) (Botys)

plenistigmalis (Warren, 1895) (Pachybotys)

pluto (Butler, 1887) (Omiodes)

plutusalis Walker, 1859

punctiferalis (Guenee, 1854) (Astura)

nigralis Warren, 1896

pyrrhalis (Walker, 1859) (Zebronia)

recurrens Moore

revidata Fabricius, 1787

rigidalis (Snellen, 1890) (Zebronia)

xuthusalis (Walker, 1859) (Botys)

zebralis Moore, 1867

Diplopseustis Meyrick, 1884

constellata Warren, 1896

pallidalis Warren, 1896

periseresalis (Walker, 1859) (Ambia?)

Discothyris Warren, 1895

ferruginata Moore

megalophalis Hampson, 1899

vestigialis Snellen, 1890

Duzulia Amsel, 1952

subhyalinalis Hampson, 1900

Dysallacta Lederer, 1863

negatalis (Walker, 1859) (Phalangiodes)
Ecpyrrhorrhoe Hubner, 1825

diffusalis (Guenee, 1854) (Botys)

Epipagis Hubner, 1825

enderythralis (Hampson, 1899) (Sameodes)

militochristalis (Hampson, 1896) (Sameodes)

peritalis Walker,

pictalis (Swinhoe, 1896) (Sameodes)

Epiparbattia Caradja, 1925

pamirensis (Arora \& Mandal, 1974) (Dausara)

gloriosalis Caradja, 1925

gloriosalis whalleyi Munroe \& Mutuura, 1971

Euclasta Lederer, 1855

defamatalis (Walker, 1859) (Ilurgia)

filigeralis Lederer, 1863

Eurrhyparodes Snellen, 1880

bracteolalis (Zeller, 1852) (Botys)

plumbeimarginalis Hampson, 1898

tricoloralis (Zeller, 1852) (Botys)

Eutectona Wang \& Sung, 1980 machoeralis (Walker, 1859) (Scopula)

Filodes Geuenee, 1854

bilinealis Hampson, 1908

fulvidorsalis (Hubner, 1832) (Pinacia)

sexpunctalis Snellen, 1890

Furcivena Hampson, 1896

strigiferalis Hampson, 1896

Glyphodes Guenee, 1854

actorionalis Walker, 1859

badialis (Walker, 1859) (Botys)

bicolor Swainson, 1821

bivitralis Guenee, 1854

caesalis Walker, 1859

callizona Meyrick, 1894

canthusalis Walker, 1859

crithealis Walker, 1859

dysallactalis Hampson, 1896

ernalis Swinhoe, 1894

minimalis Hampson, 1896

orbiferalis Hampson, 1896

prothymalis Swinhoe, 1892

proximalls Snellen, 1899

pulverulentalis Hampson, 1896

pyloalis Walker, 1859

stolalis Guenee, 1854

Goniorhynchus Hampson, 1896

flaviguttalis Warren, 1896

gratalis (Lederer, 1863) (Botys)

Herpetogramma Lederer, 1863

bipunctalis (Fabricius, 1794) (Phalaena)

dilatatipes (Walker, 1866) (Botys)

nigricornalis (Swinhoe) (Pachyzancla)

Heterocnephes Lederer, 1863

lubricosa (Warren, 1896 (Charitoprepes)

lymphatalis Swinhoe, 1889

Hyalobathra Meyrick, 1885

aequalis (Lederer, 1863) (Botys)

archeleuca Meyrick 1885

coenostolalis Snellen, 1880

filalis (Guence, 1854) (Asopia)

illectalis (Walker, 1859) (Botys)

miniosalis (Guenee, 1854) (Ebulea)

opheltisalis Walker, 1859

phoenicozona (Hampson, 1896) (Isocentris)

rubralis (Swinhoe, 1906) (Isocentris)

Hyaloplaga Warren, 1892

pulchralis Moore, 1867 
Hydrorybina Hampson, 1896 bicolor (Moore, 1888) (Syllythra)

Hydriris Meyrick, 1885

elutalis (Walker, 1859) (Ercta)

ornatalis (Duponchel, 1832) (Asopia)

Hymenia Hubner, 1825

perspectalis (Hubner, 1790) (Zinckenia)

Ischnurges Lederer, 1863

argentalis Hampson

gratiosalis Walker, 1859

luteomarginalis Hampson

rosea (Warren, 1896) (Thysanodesma)

rufalis (Hampson) (Ischnurges)

Lamprosema Hubner, 1823

acrobasella (Hampson, 1912) (Nacoleia)

albicilialis (Hampson, 1903) (Nacoleia)

albiflavalis Hampson, 1903

aurantifascialis (Hampson, 1896) (Nacoleia)

barbata (Warren,1896) (Preneopogon)

benepictalis (Warren, 1896) (Idiusia)

charesalis (Walker, 1859) (Botys)

commixta (Butler, 1879) (Samea)

conisata (Hampson, 1912) (Nacoleia)

cuprealis Moore, 1877

cyanealis (Walker, 1859) (Isopteryx)

discalis (Warren, 1852) (Thysanodesma)

eximialis (Warren, 1896) (Thysanodesma)

ferrestincta (Hampson, 1912) (Nacoleia)

foedalis (Guenee, 1854) (Isopteryx)

fusalis (Warren, 1896) (Thvsanodesma)

fuscifimbrialis (Hampson, 1896) (Nacoleia)

fuscifusalis (Hampson, 1912) (Nacoleia)

heliaula (Meyrick, 1894) (Metasiodes)

insolitalis (Walker, 1862) (Astura)

leucosemalis (Hampson, 1912) (Nacoleia)

major Butler

marionalis (Walker, 1859) (Desmia)

megaspilalis (Hampson, 1912) (Nacoleia)

mellealis Swinhoe, 1890

nigricostalis (Hampson, 1908) (Nacoleia)

niphealis Walker, 1859

ossea Butler

pachytornalis (Hampson, 1912) (Nacoleia)

pedicialis (Snellen, 1895) (Ercta)

phaleasalis (Walker, 1859) (Botys)

poeonalis (Walker, 1859) (Botys)

praeteritalis Walker, 1859

rosea (Warren, 1896) (Thysanodesma)

schrizonalis (Hampson, 1912) (Nacoleia)

scitalis Swinhoe, 1885

silvosalis (Swinhoe, 1906 (Pyrausta)

subargentalis (Snellen, 1890) (Botys)

tampiusalis (Walker, 1859) (Botys)

tiasalis (Walker, 1859) (Botys)

tristrialis Bremen

confusalis (Warren, 1896) (Hedylepta)

tumidicostalis (Hampson, 1908) (Nacoleia)

ustulalis (Hampson, 1903) (Nacoleia)

valvata (Warren, 1896) (Tylostega)

Lepidoneura Hampson, 1896

longipalpis Swinhoe

Leucinodes Guenee, 1854

apicalis Hampson, 1896

diaphana (Hampson, 1891) (Cirrhochrista)

orbonalis Guenee, 1854

Limbobotys Munroe \& Mutuura, 1970

limbolalis (Moore, 1877) (Crocidophora)

Loxoneptera Hampson, 1896

carnealis Hampson, 1896
Loxostege Hubner, 1825

brevivittalis (Hampson, 1896 (Phlyctaenodes)

crocalis (Hampson) (Phlyctaenodes)

nubilalis Hampson

oculifera (Hering, 1901) (Eurycreon)

palmalis Swinhoe, 1884

perbonalis (Swinhoe, 1890) (Phlyctaenodes)

rubralis (Warren, 1896) (Eurycreon)

simplalis (Swinhoe, 1889) (Phlyctaenodes)

vespeprtilio (Warren, 1896) (Plateopsis)

Luma Walker, 1863

flavalis Hampson

flavimarginalis Hampson, 1907

longidentalis Hampson, 1903

monomma (Warren, 1896) (Cyclarcha)

obscuralis (Swinhoe, 1895) (Loxocorys)

ornatalis Leech

sericea Butler

unicolor Moore

Lygropia Lederer, 1863

amplificata (Warren, 1896) (Metoeca)

amyntusalis (Walker, 1859) (Botys)

distorta Moore

euryclealis Walker, 1859

flavicaput Warren, 1895

flavispila Swinhoe

orbinusalis (Walker, 1859) (Astura)

pottisalis (Walker, 1859) (Botys)

shevaroyalis Hampson, 1908

Mabra Moore, 1885

daulialis (Warren, 1896) (Neurophruda)

eryxalis (Walker, 1859) (Asopia)

fuscipennalis Hampson, 1897

nigriscripta Swinhoe, 1895

Macaretaera Meyrick, 1886

hesperis Meyrick, 1886

Macrobela Turner, 1939

plumbealis (Warren, 1896) (Syntomodora)

Cnaphalocrocis Lederer, 1863

medinalis (Guenee, 1854) (Salbia)

Marasmia Lederer, 1863

bilinealis (Hampson, 1891) (Dolichosticha)

euryterminalis Hampson, 1917 (Marasmia)

fusifascialis Hampson, 1896 (Marasmia)

latimarginalis (Hampson, 1891) (Dolichosticha)

patnalis Bradley, 1981 (Marasmia)

pilosa (Warren, 1896) (Lasiacme)

mimica (Warren, 1896) (Lasiacme)

poeyalis (Boisduval, 1833) (Botys)

ruralis (Walker, 1859) (Botys)

suspicalis (Walker, 1859) (Botys)

trapezalis (Guenee, 1854) (Salbia)

trebiusali (Walker, 1859) (Botys)

Margarochroma Warren, 1896

pictalis Warren, 1896

Maruca Walker, 1859

amboinalis Felder

testulalis Geyer, 1832

Massepha Walker, 1859

absolutalis Walker, 1859

ambialis Hampson, 1903

bengalensis Moore

carbonalis Warren

rufescens Hampson, 1912

Meroctena Lederer, 1863

pullalis 1859
Metasia Guenee, 1854

annuliferalis Hampson, 1903

sabulosali Warren, 1896

straminealis Hampson, 1903

ustalis Hampson

Micromania Swinhoe, 1894

stigmatilis Swinhoe, 1894

Nagiella Munroe, 1976

hortulatoides Munroe, 1976

quadrimaculalis (Kollar, 1844) (Scopula)

Nausinoe Hubner, 1825

geometralis (Guenee, 1854) (Lepyrodes)

pueritia (Cramer, 1780) (Phalaena)

ejectata (Fabricius, 1775) (Phalaena)

Neadeloides Bryk, 1939

cinerealis (Moore, 1867) (Adeloides)

glaucoptera (Hampson, 1896) (Adeloides)

Nevrina Guenee, 1854

procopia Cramer

Niphostola Hampson, 1896

micans Hampson, 1896

Nomophila Hubner, 1825

noctuella (Denis \& Schiffermuller, 1775) (Tinea)

Noorda Walker, 1859

blitealis Walker, 1859

Nosophora Lederer, 1863

albiguttalis Swinhoe, 1890

althealis (Walker, 1859) (Botys)

conjunctalis Walker, 1865

chironalis (Walker, 1859) (Botys)

dispilalis Hampson, 1896

mesosticta Hampson, 1912

obliqualis Hampson

parvipunctalis Hampson, 1896

scotaula Meyrick, 1894

semitritalis Lederer, 1863

triguttalis Warren, 1896

Notarcha Meyrick, 1884

quaternalis (Zeller, 1852) (Botys)

Oligocentris Hampson, 1896

deciusalis (Walker, 1859) (Botys)

Omiodes Guenee, 1854

diemenalis (Guenee, 1854) (Asopia)

indicata (Fabricius, 1775) (Phalaena)

noctescens (Moore) (Charema)

surrectalis (Walker, 1866) (Deba)

Omphisa Moore, 1886

anastomosalis Guenee, 1854

fuscidentalis (Hampson, 1895) (Chilo)

repetitalis Snellen, 1890

Oronomis Munroe \& Mutuura, 1968

xanthothysana (Hampson, 1899) (Pyrausta)

Orphnophanes Lederer, 1896

laevalis (Warren, 1896) (Parapoynx)

Orthoraphis Hampson, 1896

metasticta Hampson, 1898

obfuscata Hampson, 1893

Ostrinia Hubner, 1825

appositalis (Lederer, 1858) (Botys)

dorsivittata (Moore, 1888) (Hapalia)

scapulalis assamensis Mutuura \& Munroe, 1970

zealis (Guenee, 1854) (Botys) 
Pagyda Walker, 1859 amphisalis Walker, argyritis Hampson, 1899 auroralis Moore

botydalis (Snellen, 1880) (Glyphodes) discolor Swinhoe

exalbalis Hampson, 1896

fulvistriga Swinhoe

Synclera Lederer, 1863

himachalensis Pajni \& Rose, 1978

Iustralis Snellen, 1890

salvalis Walker, 1859

straminealis Hampson, 1896

subtessellalis (Walker, 1865) (Botys)

univocalis (Walker, 1859) (Glyphodes)

Palpita Hubner, 1808

annulata (Fabricius, 1794) (Phalaena)

fraterna Moore

nigropunctalis (Brem, 1864) (Margarodes)

picticostalis Hampson, 1896

unionalis Hubner, 1796) (Pyralis)

warrenalis (Swinhoe, 1894) (Margaronia)

Parbattia Moore, 1888

serrata (Munroe \& Mutuura, 1971)

vialis Moore, 1888

Parotis Hubner, 1831

athysanota Hampson, 1912

fallacialis (Snellen, 1890) (Margaronia)

marginata (Hampson, 1893) (Cenoneimis)

marinata (Fabricius, 1794) (Phalaena)

nilgirica (Hampson, 1854) (Margarodes)

pononalis (Guenee, 1854) (Margarides)

punctiferalis (Walker, 1896) (Margaronia)

suralis (Lederer, 1863) (Chloauges)

vertumnalis (Guenee, 1854) (Margarodes)

Perisyntrocha (Meyrick, 1894)

ossealis Hampson, 1896

Phlyctaenomorpha Amsel, 1970

euralis (Hampson, 1903) (Pyrausta)

Phostria Hubner, 1819

analis Snellen, 1880

chrysalis (Hampson, 1908) (Phryganodes)

crithonalis (Walker, 1859) (Botys)

discipunctalis (Hampson, 1903) (Phryganodes)

eradicalis (Hampson, 1908) (Phryganodes)

flocculentalis (Hampson, 1898) (Phryganodes)

glyphodalis (Walker, 1865) (Analtes)

longipennis (Warren, 1896) (Charema)

lophophoralis Hampson, 1896

maculicostalis Hampson

nicoalis (Walker, 1859) (Botys)

obscurata Moore, 1886

pachycraspedalis Hampson, 1896

palliventralis (Snellen, 1890) (Omiodes)

phaennisalis (Walker, 1859) (Botys)

schediusalis (Walker, 1859) (Botys)

stygialis (Hampson, 1912) (Phryganodes)

unitalis Guenee, 1854

unitinctalis Hampson, 1896

Physematia Lederer, 1863

concordalis Lederer, 1863

Piletocera Lederer, 1863

aegimiusalis (Walker, 1859) (Desmia)

albilunata Warren, 1896

chrysorycta (Meyrick, 1884) (Semioceros)

concisalis Walker, 1859

costipunctata (Warren, 1896) (Danaga)

discalis Hampson, 1903

discisignalis Hampson, 1917 elongalis (Warren, 1896) (Thysanodesma)

flavidiscalis Hampson, 1917

flexiguttalis Warren, 1896

maculifrons Hampson, 1917

metchrealis Hampson, 1917

nudicornis Hampson, 1897

octosemalis Hampson, 1896

Pilocrocis Lederer, 1863

barcalis (Walker, 1859) (Botys)

milvinalis (Swinhoe, 1885) (Deba)

Pionea Guenee, 1845

albactalis (Walker, 1859) (Botys)

aureolalis (Lederer, 1863) (Botys)

brevialis Walker, 1859

bryophilalis Hampson, 1903

damastesalis (Walker, 1859) (Scopula)

flavicilialis (Snellen, 1890) (Crocidophora?)

flavicinctalis (Snellen, 1890) (Crocidophora?)

forficalis Linnaeus

fuscizonalis Hampson, 1896

gracilis Warren, 1888

leuanalis Swinhoe, 1890

longipalpis Hampson, 1903

nigrostigmalis (Warren, 1896) (Udea)

octonalis (Snellen, 1890) (Botys)

poenicistis Hampson, 1896

poliosticta Hampson, 1903

praepandalis (Snellen, 1890) (Botys)

pulverulenta (Hampson, 1918) (Hapalia)

renalis Moore

scopicalis Hampson, 1908

Placosaris Meyrick 1897

callistalis (Hampson, 1896) (Pachyzancla)

coorumba (Hampson, 1891) (Opsibotys)

dohertyi Munroe \& Mutuura, 1970

intensalis (Swinhoe, 1894) (Ebulea)

lindgreni Munroe \& Mutuura, 1970

ochripunctalis (Warren, 1896) (Ebulea)

steelei Munroe \& Mutuura, 1970

swanni Munroe \& Mutuura, 1970

udealis Munroe \& Mutuura, 1970

ustulalis (Hampson, 1896) (Pachyzancla)

Pleonectoides Hampson, 1891

vinacea Hampson

Pleuroptya Meyrick, 1890

balteata (Fabricius, 1978) (Phalaena)

deficiens (Moore, 1886) (Coptobasis)

iopasalis Walker, 1859

punctimarginalis Hampson, 1896

ruralis (Scopoli, 1763) (Phalaena)

scinisalis (Walker, 1859) (Sylepta)

scinisalis varrestrictalis (Snellen, 1890) (Botys)

ultimalis (Walker, 1859) (Botys)

Polygrammodes Guenee, 1854

fuscilallis (Hampson, 1891) (Pachynoa)

hypsalis Hampson, 1896

mineusalis Walker, 1859

nigrilinealis Hampson, 1903

pectinicornalis Guenee, 1854

sabelialis Guenee, 1854

Polythlipta Lederer, 1863

cerealis Lederer, 1863

divaricata Moore

uroalis (Swinhoe, 1889) (Nausinoe)

inconspicua Moore

macralis Lederer, 1863

ossealis Lederer, 1863

peragrata Moore,

Prionopaltis Warren, 1892

sericea Warren, 1892
Proconica Hampson, 1899

nigrocyanalis Hampson, 1898

Prooedema Hampson, 1896

inscisalis (Walker, 1866) (Botys)

Prophantis Warren, 1896

octoguttalis Felder, 1874

Prorodes Swinhoe, 1894

mimica Swinhoe, 1894

Protrigonia Hampson, 1896

zizanialis Swinhoe, 1885

Psara Snellen, 1875

basalis (Walker, 1865) (Botys)

coptobasalis (Hampson, 1899) (Pachyzancla)

cynaralis (Walker, 1859) (Botys)

latifuscalis (Hampson, 1899) (Pachyzancla)

marginalis (Warren, 1896) (Goniorhynchus)

ochrifuscallis (Warren, 1896) (Hedylepta)

rufescentalis (Hampson, 1896) (Pachyzancla)

stultalis (Walker, 1859) (Botys)

subdentalis (Swinhoe) (Pachyzancla)

velitaris Meyrick, 1936

Pseudebulea Butler, 1881

fentoni Butler, 1881

Pycnarmon Lederer, 1863

abraxallis Walker, 1865

aeriferalis (Moore, 1877) (Conchylodes)

alboflavalls (Moore, 1879) (Conchylodes)

cribrata (Fabricius, 1794) (Phalaena)

dryocentra (Meyrick, 1933) (Entephria)

jaguaralis (Guenee, 1854) (Spilomela)

lactiferalis Walker, 1859

marginalis (Snellen, 1890) (Conchylodes?)

meritalis (Walker, 1859) (Zebronia)

nebulosalis Hampson, 1896

radiata (Warren, 1896) (Aripana)

virgatalis Moore, 1867

Pygospila Guenee, 1854

costiflexalis Guenee, 1854

cuprealis (Swinhoe, 1892)

octomaculalis Moore, 1867

tyres (Cramer, 1780) (Phalaena Pyralis)

Pyrausta Schrank, 1802

acutidentalis Hampson, 189

atrifusalis Hampson, 1903

aurantifascialis Hampson, 1896

bambucivora Moore

bambusalis Moore

benenotata Swinhoe

bisignata Butler, 1889

bitincta Meyrick, 1932

canotinctalis Hampson, 1896

celatalis (Walker, 1859) (Botys)

cespitalis Schiffermuller

ciniferalis (Walker, 1865) (Botys)

oitrinalis Warren, 1892

cruoralis (Warren, 1895) (Syllathria)

deductalis (Walker, 1859) (Botys)

diniasalis Walker, 1859

discipunctalis (Caradja, 1935) (Pyrausta)

eriopisalis (Walker, 1859) (Botys)

euprepialls Hampson, 1903

euryphaea Meyrick, 1932

extinctalis Christoph

ferrifusalis Hampson, 1896

foviferalis Hampson, 1896

fraudulentalis (Warren, 1895) (Spliodes)

fuscalis (Warren, 1896) (Metasia)

indistans Moore,

infuscalis Hampson, 1918 
kenalis Swinhoe, 1900

metasialis Hampson, 1912

microdontalis Hampson, 1912

minimalis Hampson, 1903

monosema Hampson, 1912

nigrescens Moore

nigritalis Hampson, 1896

obliquata Moore

ochracealis Walker, 1865

panopealis (Walker, I859) (Rhodaria)

paupellalis (Lederer, 1863) (Botys)

pboenicealis Hubner, 1818

phragmatidalis Hampson, 1908

profusalis (Warren, 1896) (Opsibotys)

purpurascens Hampson

robusta Moore

rubellalis (Snellen, I890) (Botys)

rubritinctalis Hampson, 1919

rubritinctalis (Warren, 1895) (Syllythira)

sanguinalis (Linnaeus, 1767) (Pyralis)

sikkima Moore

silhetalis Guenee

simplex (Warren, 1896), (Ebuleodes)

testalis (Fabriius, 1794) (Phalaena)

vitellinalis Kolla

Ravanoa Moore, 1885

xiphialis (Walker, 1859) (Zebronia)

Rehimena Walker, 1866

hypostictalis Hampson, 1908

phrynealis (Walker, 1859) (Botys)

stictalis Hampson, 1908

striolalis (Snellen, 1890) (?Filodes)

surusalis (Walker, 1859) (Botys)

vilialis (Swinhoe, 1906)

Rhimphalea Lederer, 1863

ochalis Walker

trogusalis (Walker, 1859) (Botys)

Rhimphaleodes Hampson, 1893

macrostigma Hampson, 1893

Rodaba Moore, 1888

angulipennis Moore, 1888

Sameodes Snellen, 1880

cancellalis (Zeller, 1852) (Botys)

Sinibotys Munroe \& Mutuura, 1969

evenoralis (Walker) (Crocidophora)

Sisyrophora Lederer, 1863

pfeifferae (Lederer, 1863) (Glyphodes)

Sitochora Hubner, 1825

palealis Denis \& Schiffermuller, 1775

verticalis (Linnaeus)

Spoladea Guenee, 1854

recurvalis (Fabricius, 1775) (Phalaena)

Stemorrhages Lederer, 1863

amphitritalis (Guenee, 1854) (Margarodes)

marthesiusalis (Walker, 1859) (Margaronia)

Stenia Guenee, 1845

carbonalis (Warren, 1896) (Stenia)

bruguiralis (Duponchel, 1831) (Botys)

minoralis (Snellen, 1880 (Auxomitia)

paediusalis Walker, 1858

punctalis (Denis \& Schiffermuller, 1775)

rubriceps Hampson, 1903

Sufetula Walker, 1859

nitidalis Hampson, 1908

rectifascialis Hampson, 1896 sunidesalis Walker, 1859

Syllepte Hubner, 1823

adductalis (Walker, 1859) (Sylepta)

agraphalis Hampson, 1912

angustalis (Snellen, 1890) (Botys)

bipunctalis (Warren, 1888) (Samea?)

carbatinalis (Swinhoe, 1890) (Pramadea)

chalybifascia Hampson, 1896

chromalis Walker, 1865

cohaesalis (Walker, 1865) (Botys)

cometa (Warren, (Sylepta)

concatenalis (Walker, 1865) (Rhodaria)

costalis (Moore, 1887) (Botyodes)

crotonalis (Walker, 1859) (Syiepta)

denticulata (Moore, 1888) (Pramadea)

derogata (Fabricius, 1775) (Phalaena)

fraterna Moore, 1885) (Coptobasis)

gastralis (Walker, 1865) (Glyphodes)

lactiguttalis (Warren, 1896) (Arthriobasis)

luctuosalis (Guenee, 1854) (Sylepta)

lunalis (Guenee, 1854) (Botys)

microsema Hampson, 1912

mysissalis Walker, 1859

nigriflava Swinhoe, 1894

ochrotichroa Hampson, 1918

ogoalis (Walker, 1859) (Botys)

paucistrialis (Warren, 1896) (Cyclarcha)

penthodes (Meyrick, 1902) (Notarcha)

pernitescens (Swinhoe, 1894) (Charema)

picalis Hampson, 1898

pronamalis (Walker, 1859) (Botys)

pseudovialis Hampson, 1912

sabinusalis (Walker, 1859) (Botys)

sellalis Guenee, 1854

seminigralis (Warren, 1896) (Polycorys)

stigmatalis (Warren, 1896) (Notarcha)

straminalis (Guenee, 1854) (Salbia)

syngonias Meyrick, 1939

subalbidalis (Swinhoe, 1894) (Charema)

textalis Lederer, 1863

tibialis Moore, 1887

venustalis Swinhoe, 1894

verecunda (Warren, 1896) (Loxoscia)

violaceotinota Caradja, 1939

Symphonia Hampson, 1896

multipictals Hampson, 1896

Syngamia Guenee, 1854

abruptalis (Walker, 1859) (Asopia)

angustale (Hampson, 1893) (Orphanostigma)

falsidicalis (Walker, 1859) (Asopia)

latimarginalis Walker, 1859

oggalis (Swinhoe, 1906) (Platamonia)

vibiusalis Walker

violata Fabricius

Tabidia Snellen, 1880

aculealis (Walker, 1865) (Botys)

candidalis (Warren, 1896) (Aripana)

fuscifusalis (Hampson, 1917) (Tabidia)

inconsequens (Warren, 1896) (Nymphula)

Talanga Moore, 1885

sexpunctalis (Moore, 1877) (Oligostigma)

Tangla Swinhoe, 1900

zangisalis (Walker, 1859) (Glyphodes)

Tatobotys Butler, 1880

janapalis (Walker, 1859) (Botys)

Terastia Guenee, 1854

egialealis (Walker, 1859) (Megaphysa)

meticulosalis Guenee, 1854

Tetridia Warren, 1890 caletoralis (Walker, 1859) (Botys)

Thliptooeras Warren, 1890

cascalis Swinhoe, 1890

decoloralis (Warren, 1896) (Ebulea)

distictalis Hampson, 1899

epicrocalis Swinhoe

fulvimargo (Warren, 1895) (Mimocomma)

stygiale Hampson, 1896

triplagalis (Warren, 1896) (Prophantis)

Thysanoidma Hampson, 1891

octalis Hampson, 1891

Torqueola Swinhoe, 1906

ophiceralis Walker, 1865

Tyspanodes Warren, 1891

cardinalis Hampson, 1896

fascialis Moore, 1867

flaviventer 1891

linealis (Moore, 1867) (Propachys)

nigrolinealis Moore, 1867

Udea Guenee, 1845

ferrugalis Hubner, 1796

Ulopeza Zeller, 1852

idyalis (Walker, 1859) (Botys)

semivialis Moore

Uresiphita Hubner, 1825

dissipatalis (Lederer 1863) (Botys)

polygonalis (Denis \& Schiff Mu, 1775) (Pyralis)

orientalis (Fabricius, 1794) (Pyralis)

Xanthomelaena Hampson, 1896

schematias (Meyrick, 1894) (Tylostega)

Hampson, G.F. (1903). The moths of India. Supplementary paper to the volumes on the "Fauna of British India" Series II. Part VIII, IX and X. Journal of the Bombay Natural History Society 15: 19-37, 206-226, 650-653.

Hampson, G.F. (1906). On new Thyrididae and Pyralididae. Annals and Magazine of Natural History, London 17 (7) 189-222.

Hampson, G.F. (1908). The moths of India. Supplementary paper to the volumes of the "Fauna of British India" Series III Parts X and XI. Journal of the Bombay Natural History Society 18: 257271, 572-585.

Hampson, G.F. (1910). The moths of India. Supplementary paper to the volumes on the "Fauna of British India". Series IV. Part I. Journal of the Bombay Natural History Society 20: 83-125.

Hampson, G.F. (1912a). The moths of India. Supplementary paper to the volumes on the "Fauna of British India". Series IV. Part V. Journal of the Bombay Natural History Society 221: 411-446.

Hampson, G.F. (1912b). Descriptions of new species of Pyralidae of the sub family Pyraustinae. Annals and Magazine of Natural History, London 9(8): 149-174.

Hampson, G.F. (1912c). Descriptions of new species of Pyralidae of the sub family Pyraustinae. Annals and Magazine of Natural History, London, 9(8): 242-269.

Hampson, G.F. (1912d). Descriptions of new species of Pyralidae of the sub family Pyraustinae. Annals and Magazine of Natural History, London 9(8): 321-336. 
Hampson, G.F. (1912e). Descriptions of new species of Pyralidae of the sub family Pyraustinae. Annals and Magazine of Natural History, London 9(8): 433-444.

Hampson, G.F. (1912f). Descriptions of new species of Pyralidae of the sub family Pyraustinae. Annals and Magazine of Natural History, London 9(8): 557-573.

Hampson, G.F. (1912g). Descriptions of new species of Pyralidae of the sub family Pyraustinae. Annals and Magazine of Natural History, London 9(8): 625-633.

Hampson, G.F. (1912h). Descriptions of new species of Pyralidae of the sub family Pyraustinae. Annals and Magazine of Natural History, London 10(8): 1-20.

Hampson, G.F. (1913a). Descriptions of new species of Pyralidae of the sub family Pyraustinae. Annals and Magazine of Natural History, London 2(8): 1-38

Hampson, G.F. (1913b). Descriptions of new species of Pyralidae of the sub family Pyraustinae. Annals and Magazine of Natural History, London 11 (8): 322-342.

Hampson, G.F. (1913c). Descriptions of new species of Pyralidae of the sub family Pyraustinae. Annals and Magazine of Natural History, London 9 (8): 509-530.

Hampson, G.F. (1917a). A classification of the Pyralidae Subfamily, Hypsotropinae, Proceedings of Zoological Society of London Ser. 8, 19: 55-131.

Hampson, G.F. (1917b). Descriptions of new Pyralidae of the subfamilies Epipaschiinae, Chrysauginae, Endotrichinae and Pyralinae. Annals and Magazine of Natural History, London Ser. 8, 19: 65-100.

Hampson, G.F. (1917c). Descriptions of new Pyralidae of the subfamilies Hydrocampinae and Scorpariinae. Annals and Magazine of Natural History, London Ser. 8, 20: 201-216; 265-82.

Hampson, G.F. (1917d). A classification of the Pyralidae subfamily, Gallerianae. Novitates Zoologicae 225: 17-58.

Hampson, G.F. (1917e). Descriptions of new Pyralidae of the subfamilies Crambinae and Siginae. Annals and Magazine of Natural History, London, Ser. 9, (20), Vol.4:53-68:137-154; 305-326; 275-292; 437-455; 533-547. Hampson, G.F. (1930). New genera and Species of Phycitinae (Lep., Pyralidae). Annals and Magazine of Natural History, London, Ser., 10(5): 50-80.

Mathew, G. and M.G.R. Menon (1984). The pyralid fauna of Kerala. $J l$. Ent. Res. 8(1): 5-13.

Mathew, G. and M.G.R. Menon (1985). External genitalia of some Indian pyralids. Journal of Entomological Research 9(1) : 26-35.

Mathew, G. and M.G.R. Menon (1986). Identification of some leaf rollers belonging to the genera Bradina, Marasmia and Cnphalocrosis (Lepidoptera: Pyraustidae). Entomon 11(4): 311-317.

Mathew, G. (1987). Biosystematics in Lepidoptera and its importance in forest entomological research. Proceedings of the Indian Academy of Science (Animal Science) 96(5): 613-616.

Mathew, G. and M.G.R. Menon (1988). Identification of some Indian Pyaustinae. Entomon 13 (1): 75-90.

Mathew, G. and M.G.R. Menon (1989). Identification of some Indian Pyrastinae. Journal of Entomological Research 13(1): 76-89.

Moore, F. (1865). Proceedings of Zoological Society of London p814, p.43.

Moore, F. (1867). Proceedings of Zoological Society of London 87-96.

Moore, F. (1879-1888). Descriptions of new Indian lepidopterous insects from the collection of W.S. Atkinson, Heterocera, Calcutta.

Moore, F. (1879). Scientific results of the 2nd Yarkand Mission. Lepidoptera, Calcutta.

Moore, F. (1882-1887). The Lepidoptera of Ceylon, Heterocera, London, Vols. II \& IV.

Munroe, E. and A. Mutuura (1970). Contribution to a study of the Pyraustinae, (Lep., Pyralidae) of temperate East Asia, X, Can. Ent., 101: 1489-1507.

Munroe, Eugene (1972). The Moths of North America, Fascicle 13.1A 12-14pp.

Munroe, Eugene and Solis, Alma (1999). The Pyraloidea. pp.233-256. In: Kristensen, N.P. (Ed.). Handbook of Zoology, Section Lepidoptera:
Moths and Butterflies. Walter de Gruyter, New York.

Roesler, U. (1969). Phycitinen Studien-VIII. Ent. Ztschr., 79:396-407.

Rose, H.S., (1981). Description of a new galleriid, Lamoria hemi (Lepidoptera, Galleriidae, Pyralidae) from North India. Entomon 6(2): 167171.

Rose, H.S. and J. Singh (1992). Studies on the Indian species of the genus Palpita Hubner (Lepidoptera: Pyraustinae). Journal of Entomological Research 16(1): 62-77.

Rose, H.S. and J. Singh (1999). Taxonomic studies on 3 species genus Botyodes (Pyraustinae: Pyralidae). Journal of Institution of Science 3(2):118121.

Rose, H.S. and J. Singh (1989). Further studies on genus Glyphodes Guen. (Pyraustinae: Lepidoptera). Proceedings NMYS: 195-200.

Snellen, P.C.T. (1882). D'Vlinders Van Nederland Microlepidoptera, I Leiden, 13, $536 \mathrm{pp}$.

Snellen, P.C.T. (1890). A catalogue of the Pyralidinae of Sikkim Collected by Henry J.E. Elwes and Late Otto Moller, with notes by Elwes. Transactions of the Entomological Society of London, pp., 557-647, pls., 14, 20

Swinhoe, C. (1884). On the Lepidoptera collected from Kurrachee. Proceedings of the Zoological Society of London, 528pp.

Swinhoe, C. (1885). On the Lepidoptera of Bombay and Deccan. Proceedings of the Zoological Society of London, 879pp.

Swinhoe, C. (1889a). Proceedings of the Zoological Society of London, 418-422.

Swinhoe, C. (1889b). Proceedings of the Zoological Society of London, 1153-479

Swinhoe, C. (1892). New species of Heterocera from Khasya Hills, pt .I, Transactions of the Entomological Society of London pp. 473-495, pl. 15. Swinhoe, C. (1894). New Species of Eastern Lepidoptera, Annal of Natural History 6(14): 135-149; 429-443.

Swinhoe, C. (1900). Catalogue of Eastern and Australian Lepidoptera Heterocera in the collection of the Oxford University Museum. Part I. Noctuina, Geometrina and Pyralidina, Oxford.

Swinhoe, C. (1906, 1907). New and little known Species of Heterocera from the East. Annals and Magazine of Natural History 7(17):283-297.

Swinhoe, C. (1916). New Indo-Malayan Lepidoptera, Annals and Magazine of Natural History, London 18: 209-221.

Whalley, P.E.S. (1971). A Synoptic catalogue of the genera of phycitinae (Lep., Pyralidae), of the World. Entomololy 25(2): 31-72.

\section{ACKNOWLedgements}

I am profoundly grateful to late Dr. M.G. Ramdas Menon (erstwhile Emeritus Scientist, ICAR, New Delhi and my Ph.D Research Guide) for inspiring me to carry out taxonomic studies of Indian Lepidoptera. Preparation of inventories of Indian insects had been his unaccomplished dream. Although attempts were made to prepare a check list of Indian pyralids as early as 1975, it could not be accomplished due to various reasons. However, due to the persistent encouragement from my senior colleague, Dr. K.S.S. Nair (former Director, KFRI) as well as from various specialists of this group of insects like Dr. J.D.Holloway (formerly of the British Museum of Natural History, London) and Dr. Alma Solis (Smithsonian Institution, Washington), I was able to do partial justice by preparing a preliminary list of the pyralid moths of India.

The draft list prepared earlier, have been thoroughly revised taking into account the nomenclatural changes to the various taxa. For this, I have received considerable help from Mr. M. Shaffer, Lepidopterist, British Museum of Natural History, London, who allowed me to make use of many of his notes on the museum card indexes. In addition to this, he had also advised me on the nomenclatural aspects of various pyralid taxa during my period of work in the museum. I am thankful to Dr. Gaden Robinson, Dr.J.D. Bradley and Dr. K.M. Harris (erstwhile Director) of the International Institute of Entomology, London for kindly permitting me to refer to the literature as well as insect collections. I also wish to acknowledge my gratitude to the British Council for awarding me a 'visitorship' which enabled me to undertake this study. The encouragement rendered to me by Dr. J.K. Sharma (Director, KFRI) is also gratefully acknowledged.

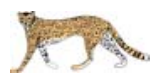

